University of Nebraska - Lincoln

DigitalCommons@University of Nebraska - Lincoln

Papers in the Earth and Atmospheric Sciences

Earth and Atmospheric Sciences, Department

September 2003

\title{
Late Quaternary lake-level changes constrained by radiocarbon and stable isotope studies on sediment cores from Lake Titicaca, South America
}

\author{
Harold D. Rowe \\ Geological and Environmental Sciences, Stanford University, BIdg. 320, Rm. 118, Stanford, CA \\ Thomas P. Guilderson \\ Center for Accelerator Mass Spectrometry, Lawrence Livermore National Laboratory, Livermore, CA \\ Robert B. Dunbar \\ Geological and Environmental Sciences, Stanford University, Bldg. 320, Rm. 118, Stanford, CA \\ John R. Southon \\ Center for Accelerator Mass Spectrometry, Lawrence Livermore National Laboratory, Livermore, CA \\ Geoffrey O. Seltzer \\ Syracuse University \\ See next page for additional authors \\ Follow this and additional works at: https://digitalcommons.unl.edu/geosciencefacpub \\ Part of the Earth Sciences Commons
}

Rowe, Harold D.; Guilderson, Thomas P.; Dunbar, Robert B.; Southon, John R.; Seltzer, Geoffrey O.; Mucciarone, David A.; Fritz, Sherilyn C.; and Baker, Paul A., "Late Quaternary lake-level changes constrained by radiocarbon and stable isotope studies on sediment cores from Lake Titicaca, South America" (2003). Papers in the Earth and Atmospheric Sciences. 25.

https://digitalcommons.unl.edu/geosciencefacpub/25

This Article is brought to you for free and open access by the Earth and Atmospheric Sciences, Department of at DigitalCommons@University of Nebraska - Lincoln. It has been accepted for inclusion in Papers in the Earth and Atmospheric Sciences by an authorized administrator of DigitalCommons@University of Nebraska - Lincoln. 


\section{Authors}

Harold D. Rowe, Thomas P. Guilderson, Robert B. Dunbar, John R. Southon, Geoffrey O. Seltzer, David A. Mucciarone, Sherilyn C. Fritz, and Paul A. Baker 


\title{
Late Quaternary lake-level changes constrained by radiocarbon and stable isotope studies on sediment cores from Lake Titicaca, South America
}

\author{
Harold D. Rowe $\mathrm{a}^{\mathrm{a}, \mathrm{b}, *}$, Thomas P. Guilderson ${ }^{\mathrm{c}, \mathrm{d}}$, Robert B. Dunbar ${ }^{\mathrm{a}}$, \\ John R. Southon ${ }^{\mathrm{c}}$, Geoffrey O. Seltzer ${ }^{\mathrm{e}}$, David A. Mucciarone ${ }^{\mathrm{a}}$, \\ Sherilyn C. Fritz ${ }^{\text {, }}$ Paul A. Baker ${ }^{\mathrm{g}}$ \\ ${ }^{a}$ Geological and Environmental Sciences, Stanford University, Bldg. 320, Rm. 118, Stanford, CA 94305-2115, USA \\ ${ }^{\mathrm{b}}$ Earth and Environmental Sciences, University of Illinois at Chicago, SES Bldg., MC-186, \\ 845 West Taylor Avenue, Chicago, IL 60607-7059, USA \\ ${ }^{\mathrm{c}}$ Center for Accelerator Mass Spectrometry, Lawrence Livermore National Laboratory, Livermore, CA 94551, USA \\ ${ }^{\mathrm{d}}$ Department of Ocean Sciences and Institute of Marine Sciences, Earth and Marine Sciences Bldg., Rm. A316, \\ University of California, Santa Cruz, Santa Cruz, CA 95064, USA \\ ${ }^{\mathrm{e}}$ Department of Earth Sciences, 204 Heroy Geology Laboratory, Syracuse University, Syracuse, NY 13244-1070, USA \\ ${ }^{\mathrm{f}}$ Department of Geosciences, 214 Bessey Hall, University of Nebraska-Lincoln, Lincoln, NE 68588-0340, USA \\ ${ }^{\mathrm{g}}$ Division of Earth and Ocean Sciences, Duke University, Box 90227, Durham, NC 27708-0227, USA
}

Received 25 November 2001; accepted 9 April 2002

\begin{abstract}
We present and compare AMS- ${ }^{14} \mathrm{C}$ geochronologies for sediment cores recovered from Lake Titicaca, South America. Radiocarbon dates from three core sites constrain the timing of late Quaternary paleoenvironmental changes in the Central Andes and highlight the site-specific factors that limit the radiocarbon geochronometer. With the exception of mid-Holocene sediments, all cores are generally devoid of macrophyte fragments, thus bulk organic fractions are used to build core chronologies. Comparisons of radiocarbon results for chemically defined fractions (bulk decalcified, humate, humin) suggest that ages derived from all fractions are generally coherent in the post-13,500 yr BP time interval. In the pre-13,500 yr BP time interval, ages derived from humate extracts are significantly younger (300-7000 years) than ages from paired humin residues. Gross age incoherencies between paired humate and humin sub-fractions in pre-13,500 yr BP sediments from all core sites probably reflect the net downward migration of humates. Ages derived from bulk decalcified fractions at our shallow water $(90 \mathrm{~m})$ and deep water $(230 \mathrm{~m})$ core sites consistently fall between ages derived from humate and humin subfractions in the pre-13,500 yr BP interval, reflecting that the bulk decalcified fraction is predominantly a mixture of humate and humin sub-fractions. Bulk decalcified ages from the pre-13,500 yr BP interval at our intermediate depth core site $(150 \mathrm{~m})$ are consistently older than humate (youngest) and humin sub-fractions. This uniform, reproducible pattern can

* Corresponding author. Department of Geological Sciences, University of Kentucky, 101 Slone Research Bldg., Lexington, KY 405060053, USA. Tel.: +1-859-257-2770; fax: +859-257-2770.

E-mail addresses: hrowe@uky.edu (H.D. Rowe), guilderson1@1lnl.gov (T.P. Guilderson), dunbar@pangea.stanford.edu (R.B. Dunbar), southon1@1lnl.gov (J.R. Southon), goseltze@mailbox.syr.edu (G.O. Seltzer),dam@pangea.stanford.edu (D.A. Mucciarone), sfritz2@unl.edu (S.C. Fritz), pbaker@geo.duke.edu (P.A. Baker).
\end{abstract}


be explained by the mobilization of a relatively older organic sub-fraction during and after the re-acidification step following the alkaline treatment of the bulk sediment. The inferred existence of this 'alkali-mobile, acid-soluble' subfraction implies a different depositional/post-depositional history that is potentially associated with a difference in source material. While internally consistent geochronologies can be developed for the Lake Titicaca sequence using different organic fractions, mobile organic sub-fractions and fractions containing mobile sub-fractions should generally be avoided in geochronology studies. Consequently, we believe humin and/or bulk decalcified ages provide the most consistent chronologies for the post-13,500 yr BP interval, and humin ages provide the most representative ages for sedimentation prior to $13,500 \mathrm{yr} \mathrm{BP}$ interval.

Using the age model derived from the deep water core site and a previously published isotope-based lake-level reconstruction, we present a qualitative record of lake level in the context of several ice-core records from the western hemisphere. We find the latest Pleistocene lake-level response to changing insolation began during or just prior to the Bølling/Allerød period. Using the isotope-based lake-level reconstruction, we also find the $85-\mathrm{m}$ drop in lake level that occurred during the mid-Holocene was synchronous with an increase in the variability of ice-core $\delta^{18} \mathrm{O}$ from a nearby icecap, but was not reflected in any of the polar ice-core records recovered from the interior of Antarctica and Greenland. (C) 2003 Published by Elsevier B.V.

Keywords: Paleoclimate; Radiocarbon; Lake sediments; Humin; Humate; South America

\section{Introduction}

Accurate age models are vitally important for the identification of leads and lags in paleoclimate records (Pilcher, 1991; Lowe and Walker, 2000). Although studies of late Quaternary lacustrine facies rely almost exclusively on the AMS- ${ }^{14} \mathrm{C}$ method for age control, most studies do not incorporate the large number of radiocarbon measurements that are necessary to address changing reservoir-water effects and constrain the timing of changes in sedimentation rates (Lowe, 1991, and references therein; Hammarlund et al., 1997). Attempts to quantify and correct for uncertainties must compare the age results of chemically and/or physically separable fractions (macrophytes, pollen, bulk organic matter, humate, and humin) from discrete intervals (Shore et al., 1995; Abbott and Stafford, 1996; Van Klinken and Hedges, 1998). Barring reservoir-water effects (those effects that occur when materials have incorporated carbon from aqueous bicarbonate derived from an old source; Shotton, 1972), age coherency between isolated fractions from a discrete interval can be taken as evidence for acceptance of an age of sedimentation. Age incoherency between fractions demands a plausible explanation for the discrepancy and elimination of the "contaminating" fraction(s).

Sites with sound geochronologies and calibrated proxy records provide the most useful paleoclimate/ paleoecological information. Reconstructions of past environmental conditions from low-latitude sites provide important clues for identifying the forcing mechanisms responsible for climate change at millennial and submillennial timescales (e.g., Guilderson et al., 1994, 2001; deMenocal et al., 2000a). We present down-core AMS $-{ }^{14} \mathrm{C}$ ages for chemically defined organic fractions from Lake Titicaca $\left(16^{\circ} \mathrm{S}, 69^{\circ} \mathrm{W}\right.$; $3810 \mathrm{~m}$ asl), South America. Lake level has fluctuated by tens of meters during the last glacial/interglacial cycle, reaching a lowstand of $85 \mathrm{~m}$ below modern level during the middle Holocene (Wirrmann and de Oliveira Almeida, 1987; Mourguiart, 1990; Wirrmann et al., 1990, 1992; Wirrmann and Mourguiart, 1995; Abbott et al., 1997a,b; Mourguiart et al., 1998; Seltzer et al., 1997; Cross et al., 1999; Baker et al., 2001; Rowe et al., 2002). Although two lake-level proxy indicators (bulk organic $\delta^{13} \mathrm{C}$, diatoms) are now recognized (Baker et al., 2001; Rowe et al., 2002), a detailed geochronology for the late Quaternary has not yet been established. This paper focuses on the radiocarbon geochronology, addressing the specific challenges of using ${ }^{14} \mathrm{C}$ as a dating tool at Lake Titicaca, and presenting the isotope-based paleoenvironmental record in the context of a new age model.

The Titicaca drainage basin $\left(57,340 \mathrm{~km}^{2}\right)$ occupies the northern third of the high-altitude tectonic plateau known as the South American Altiplano (Fig. 1a). Regional moisture originates from the tropical Atlantic 


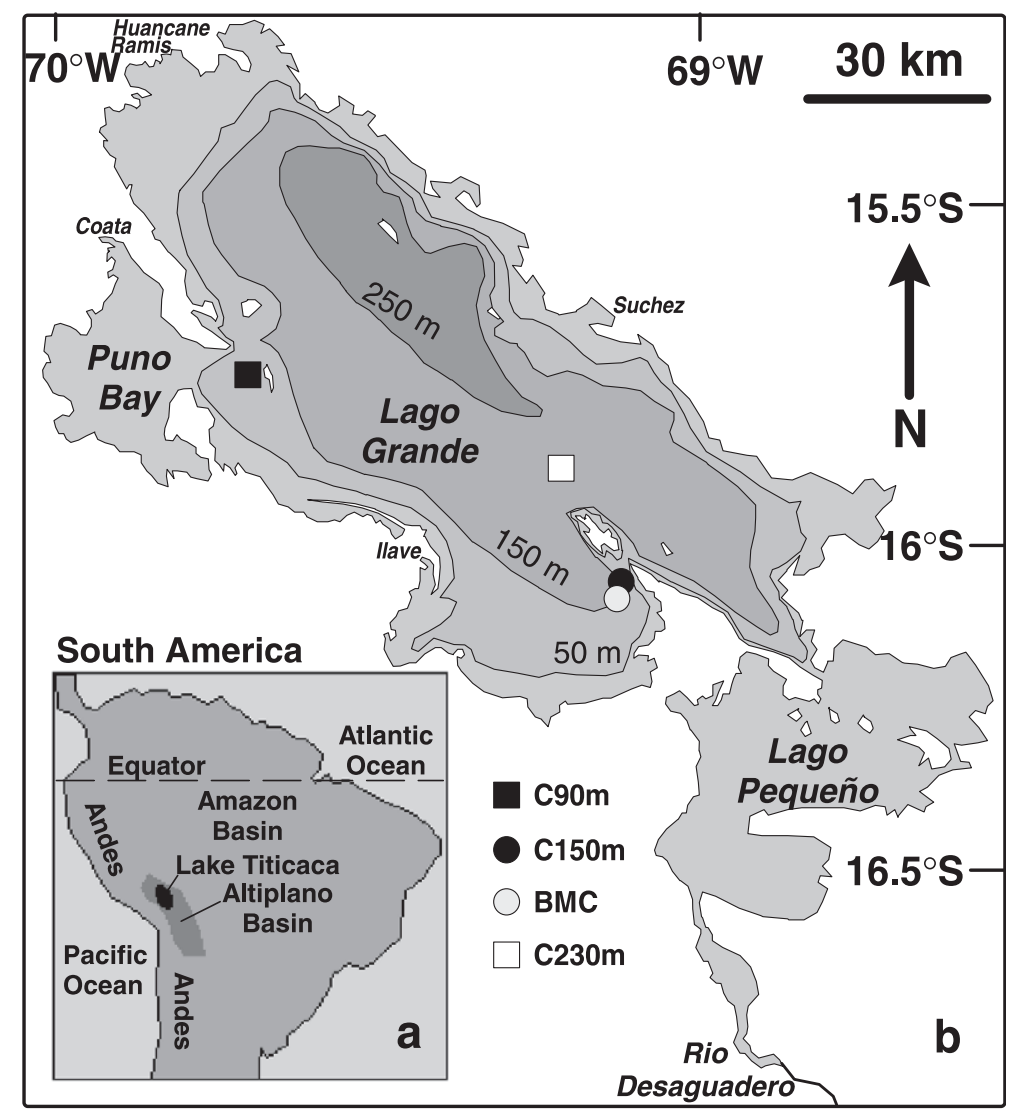

Fig. 1. (a) The location of Lake Titicaca with respect to the South American Altiplano and the Amazon Basin. (b) Map of Lake Titicaca showing core site and geographical locations. Italicized proper names illustrate inflow location of major rivers.

Ocean and is transported to the Altiplano during the austral summer (DJF) by the coupled interaction of deep convection in the Amazon Basin and middle- and upper-level easterlies associated with the Bolivian High, an interaction establishing what is now termed the South American Summer Monsoon (SASM; Zhou and Lau, 1998; Garreaud, 1999, 2000; Lenters and Cook, 1999; Vuille, 1999). Most rivers on the northern
Altiplano drain into Lake Titicaca (area $=8562 \mathrm{~km}^{2}$, volume $=903.7 \times 10^{9} \mathrm{~m}^{3}$, Wirrmann, 1992), and the lake drains to the south by way of the Rio Desaguadero. Direct evaporation accounts for $\sim 95 \%$ of the annual water loss (Monnheim, 1956). Historical lake-level changes of \pm 3 m largely reflect interannual variability of moisture transport to the northern Altiplano (Roche et al., 1992). Recent studies of the modern climate

Table 1

Location, water depth, and core length for the core sites used in this study

\begin{tabular}{|c|c|c|c|c|c|}
\hline Core site & Core & Latitude $\left({ }^{\circ} \mathrm{S}\right)$ & Longitude $\left({ }^{\circ} \mathrm{W}\right)$ & Water depth (m) & Core length $(\mathrm{m})$ \\
\hline \multirow[t]{2}{*}{$\mathrm{C} 90 \mathrm{~m}$} & NE97-7PC & $15^{\circ} 48.381^{\prime}$ & $69^{\circ} 42.096^{\prime}$ & 89 & 8.330 \\
\hline & NE98-10BXA & $15^{\circ} 48.392^{\prime}$ & $69^{\circ} 42.117^{\prime}$ & 90 & 0.970 \\
\hline \multirow{3}{*}{$\mathrm{C} 150 \mathrm{~m}$} & NE98-1PC & $16^{\circ} 08.004^{\prime}$ & $69^{\circ} 09.199^{\prime}$ & 152 & 10.600 \\
\hline & NE98-4BXB & $16^{\circ} 08.259^{\prime}$ & $69^{\circ} 09.267^{\prime}$ & 147 & 0.845 \\
\hline & $\mathrm{BMC}$ & $16^{\circ} 08.004^{\prime}$ & $69^{\circ} 09.199^{\prime}$ & 152 & 8.100 \\
\hline $\mathrm{C} 230 \mathrm{~m}$ & NE97-2PC & $15^{\circ} 52.441^{\prime}$ & $69^{\circ} 17.462^{\prime}$ & 230 & 7.865 \\
\hline
\end{tabular}


Table 2

Raw and calibrated radiocarbon results for all fractions and the corresponding sediment depths for core site $\mathrm{C} 90 \mathrm{~m}$

\begin{tabular}{|c|c|c|c|c|c|c|c|c|c|}
\hline $\begin{array}{l}\text { Laboratory } \\
\text { accession no. }\end{array}$ & $\begin{array}{l}\text { Depth below } \\
\text { sediment } \\
\text { surface }(\mathrm{cm})\end{array}$ & $\begin{array}{l}\text { Fraction } \\
\text { dated }\end{array}$ & ${ }^{14} \mathrm{C}$ age & Error & $\begin{array}{l}+1 \mathrm{~s} \mathrm{yr} \\
\mathrm{BP}\end{array}$ & $\begin{array}{l}\text { Rounded } \\
\text { yr BP } \\
\text { INTCAL98 } \\
\end{array}$ & $\begin{array}{l}-1 \mathrm{~s} y \mathrm{r} \\
\mathrm{BP}\end{array}$ & $\begin{array}{l}\text { Coral } \\
\text { calibrated } \\
\text { age yr BP }\end{array}$ & $\begin{array}{l}\text { Model age } \\
\text { yr BP }\end{array}$ \\
\hline OS-19791 & 5.0 & $\mathrm{BD}$ & 180 & 45 & 100 & 50 & 10 & - & $50^{*}$ \\
\hline OS-20624 & 50.0 & $\mathrm{BD}$ & 1020 & 30 & 710 & 680 & 670 & - & $680^{*}$ \\
\hline OS-19792 & 95.0 & $\mathrm{BD}$ & 1610 & 70 & 1310 & 1290 & 1190 & - & $1290 *$ \\
\hline OS-17702 & 176.5 & $\mathrm{BD}$ & 2630 & 25 & 2360 & 2350 & 2350 & - & $2350 *$ \\
\hline CAMS-70682 & 209.5 & $\mathrm{BD}$ & 3350 & 40 & 3360 & 3280 & 3270 & - & $3280 *$ \\
\hline CAMS-70683 & 238.5 & $\mathrm{BD}$ & 3680 & 40 & 3720 & 3660 & 3640 & - & $3660 *$ \\
\hline CAMS-70684 & 268.5 & $\mathrm{BD}$ & 4170 & 40 & 4420 & 4410 & 4300 & - & $4410^{*}$ \\
\hline CAMS-70685 & 288.5 & $\mathrm{BD}$ & 4380 & 40 & 4810 & 4620 & 4530 & - & $4620 *$ \\
\hline CAMS-70686 & 313.5 & $\mathrm{BD}$ & 4720 & 40 & 5280 & 5190 & 4980 & - & $5190 *$ \\
\hline CAMS-70687 & 338.5 & $\mathrm{BD}$ & 4850 & 40 & 5440 & 5310 & 5300 & - & $5310^{*}$ \\
\hline OS-20113 & 363.5 & $\mathrm{BD}$ & 5300 & 50 & 5890 & 5830 & 5730 & - & $5830 *$ \\
\hline OS-20628 & 424.5 & $\mathrm{BD}$ & 5630 & 60 & 6280 & 6200 & 6000 & - & $6200^{*}$ \\
\hline OS-20632 & 424.5 & $\mathrm{BC}$ & 5400 & 40 & 5930 & 5910 & 5900 & - & 5910 \\
\hline CAMS-70688 & 449.5 & $\mathrm{BD}$ & 6070 & 40 & 6720 & 6650 & 6570 & - & $6650 *$ \\
\hline CAMS-70689 & 474.5 & $\mathrm{BD}$ & 7070 & 40 & 7680 & 7670 & 7590 & - & $7670 *$ \\
\hline CAMS-70690 & 499.5 & $\mathrm{BD}$ & 7220 & 40 & 7840 & 7770 & 7740 & - & $7770^{*}$ \\
\hline CAMS-69716 & 506.0 & $\mathrm{HU}^{\S}$ & 6690 & 40 & 7420 & 7370 & 7310 & - & $7370^{*}$ \\
\hline CAMS-69717 & 506.0 & $\mathrm{HA}^{\S}$ & 7050 & 80 & 7680 & 7630 & 7580 & - & 7630 \\
\hline CAMS-70691 & 526.0 & $\mathrm{BD}$ & 7680 & 40 & 8330 & 8270 & 8180 & - & $8270 *$ \\
\hline CAMS-69850 & 536.0 & $\mathrm{HU}$ & 7800 & 40 & 8390 & 8370 & 8350 & - & $8370 *$ \\
\hline CAMS-70944 & 536.0 & HA & 8260 & 40 & 9010 & 8990 & 8780 & - & 8990 \\
\hline OS-20614 & 545.0 & $\mathrm{BD}$ & 8450 & 50 & 9270 & 9180 & 9030 & - & $9180 *$ \\
\hline CAMS-69851 & 551.0 & $\mathrm{HU}$ & 8410 & 40 & 9240 & 9090 & 9030 & - & $9090 *$ \\
\hline CAMS-69852 & 551.0 & HA & 8100 & 130 & 8980 & 8600 & 8450 & - & 8600 \\
\hline CAMS-69853 & 561.0 & $\mathrm{HU}$ & 8780 & 30 & 9530 & 9530 & 9500 & - & $9530^{*}$ \\
\hline CAMS-69893 & 561.0 & HA & 8830 & 70 & 9550 & 9540 & 9500 & - & 9540 \\
\hline CAMS-69854 & 571.0 & HU & 9190 & 40 & 10,190 & 10,150 & 9920 & - & $10,150 *$ \\
\hline CAMS-69894 & 571.0 & HA & 9300 & 70 & 10,240 & 10,220 & 10,190 & - & 10,220 \\
\hline CAMS-69855 & 581.0 & HU & 9770 & 40 & 11,060 & 11,030 & 10,690 & - & $11,030^{*}$ \\
\hline CAMS-70945 & 581.0 & HA & 10,020 & 40 & 11,200 & 11,190 & 11,170 & - & 11,190 \\
\hline OS-26866 & 586.0 & $\mathrm{BD}$ & 10,080 & 85 & 11,260 & 11,200 & 11,180 & - & $11,200^{*}$ \\
\hline CAMS-69718 & 591.0 & $\mathrm{HU}^{\S}$ & 10,100 & 40 & 11,230 & 11,230 & 11,200 & - & $11,230^{*}$ \\
\hline CAMS-69719 & 591.0 & $\mathrm{HA}^{\S}$ & 9900 & 50 & 11,170 & 11,120 & 10,810 & - & 11,120 \\
\hline CAMS-71317 & 606.0 & $\mathrm{HU}$ & 10,210 & 40 & 11,550 & 11,300 & 11,260 & - & $11,300^{*}$ \\
\hline CAMS-71318 & 606.0 & HA & 10,130 & 40 & 11,260 & 11,230 & 11,200 & - & 11,230 \\
\hline OS-26867 & 626.0 & $\mathrm{BD}$ & 10,640 & 70 & 12,620 & 12,330 & 11,970 & - & $12,330 *$ \\
\hline CAMS-71094 & 631.0 & $\mathrm{HU}$ & 10,790 & 40 & 12,830 & 12,630 & 12,350 & - & $12,630^{*}$ \\
\hline CAMS-71095 & 631.0 & HA & 10,660 & 50 & 12,630 & 12,530 & 12,110 & - & 12,530 \\
\hline OS-26868 & 675.0 & $\mathrm{BD}$ & 11,760 & 60 & 13,790 & 13,460 & 13,410 & - & $13,460 *$ \\
\hline CAMS-71096 & 683.0 & $\mathrm{HU}$ & 11,990 & 50 & 13,840 & 13,810 & 13,520 & - & $13,810^{*}$ \\
\hline CAMS-71097 & 683.0 & HA & 11,890 & 60 & 13,820 & 13,710 & 13,460 & - & 13,710 \\
\hline OS-26869 & 695.0 & $\mathrm{BD}$ & 12,610 & 60 & 15,330 & 14,320 & 14,150 & - & 14,320 \\
\hline CAMS-69720 & 718.0 & $\mathrm{HU}^{\S}$ & 13,100 & 50 & 15,680 & 15,490 & 14,910 & 15,490 & $15,490 *$ \\
\hline CAMS-69721 & 718.0 & $\mathrm{HA}^{\S}$ & 12,570 & 50 & 15,300 & 14,300 & 14,140 & - & 14,300 \\
\hline OS-20615 & 725.0 & $\mathrm{BD}$ & 13,300 & 85 & 15,930 & 15,690 & 15,390 & 15,730 & 15,730 \\
\hline CAMS-71392 & 733.0 & HU & 13,480 & 50 & 16,130 & 15,900 & 15,680 & 15,950 & $15,950 *$ \\
\hline CAMS-71098 & 733.0 & HA & 13,050 & 40 & 15,620 & 15,440 & 14,570 & 15,420 & 15,420 \\
\hline OS-20616 & 745.0 & $\mathrm{BD}$ & 14,150 & 95 & 16,940 & 16,670 & 16,430 & 16,780 & 16,780 \\
\hline OS-20116 & 760.0 & $\mathrm{BD}$ & 12,950 & 55 & 15,540 & 15,340 & 14,430 & 15,300 & 15,300 \\
\hline CAMS-69856 & 783.0 & $\mathrm{HU}$ & 15,680 & 60 & 18,730 & 18,440 & 18,160 & 18,640 & $18,640^{*}$ \\
\hline CAMS-70946 & 783.0 & HA & 13,270 & 60 & 15,890 & 15,660 & 15,340 & 15,700 & 15,700 \\
\hline
\end{tabular}


Table 2 (continued)

\begin{tabular}{llllrlllll}
\hline $\begin{array}{l}\text { Laboratory } \\
\text { accession no. }\end{array}$ & $\begin{array}{l}\text { Depth below } \\
\text { sediment } \\
\text { surface }(\mathrm{cm})\end{array}$ & $\begin{array}{l}\text { Fraction } \\
\text { dated }\end{array}$ & ${ }^{14} \mathrm{C}$ age & Error & $\begin{array}{l}+1 \mathrm{~s} \text { yr } \\
\text { BP }\end{array}$ & $\begin{array}{l}\text { Rounded } \\
\text { yr BP } \\
\text { INTCAL98 }\end{array}$ & $\begin{array}{l}-1 \text { s yr } \\
\text { BP }\end{array}$ & $\begin{array}{l}\text { Coral } \\
\text { calibrated } \\
\text { age yr BP }\end{array}$ & $\begin{array}{l}\text { Model age } \\
\text { yr BP }\end{array}$ \\
\hline CAMS-69857 & 826.5 & HU & 18,300 & 60 & 21,790 & 21,450 & 21,120 & 21,760 & $21,760^{*}$ \\
CAMS-69895 & 826.5 & HA & 13,500 & 240 & 16,280 & 15,930 & 15,570 & 15,980 & 15,980 \\
CAMS-69896 & 856.5 & HA & 13,420 & 170 & 16,130 & 15,830 & 15,530 & 15,880 & 15,880 \\
CAMS-69858 & 856.5 & HU & 18,270 & 60 & 21,760 & 21,420 & 21,090 & 21,720 & $21,720^{*}$ \\
CAMS-69859 & 896.5 & HU & 17,670 & 70 & 21,060 & 20,730 & 20,400 & 21,020 & $21,020^{*}$ \\
CAMS-69897 & 896.5 & HA & 12,060 & 470 & 14,300 & 13,820 & 13,190 & - & 13,820 \\
OS-20617 & 924.5 & BD & 17,200 & 95 & 20,520 & 20,180 & 19,860 & 20,460 & 20,460 \\
\hline
\end{tabular}

Radiocarbon ages younger than $12,620{ }^{14} \mathrm{C}$ yr BP are calibrated using INTCAL98 (Method A; Stuiver et al., 1998; Stuiver and Reimer, 1993; Stuiver and Polach, 1977). Older radiocarbon ages are calibrated using a second-order polynomial equation through the published U/Th- ${ }^{14} \mathrm{C}$ coral database (Bard et al., 1998). In the text and figures, calendar ages are denoted "yr BP". A 250 -year reservoir-water correction has been made to all dates (Abbott et al., 1997a,b).

BD: bulk decalcified; HU: humin; HA: humate; MF: macrophyte fragments; BC: bulk carbonate.

The box-core (NE98-10BXA) and piston core (NE97-7PC) that comprise core site C90m do not overlap; however, the time gap between the cores is believed to be less than a few hundred years.

${ }^{\S}$ Humin and humate dates from discrete intervals denoted in Fig. 2.

* Ages used in age model.

leave little doubt that interannual precipitation variability on the Altiplano, metered by the intensity of the SASM, is strongly influenced by El Niño-Southern Oscillation (ENSO) variability in the equatorial Pacific ocean-atmosphere system that teleconnects with the tropical Atlantic/Amazon climate system (Aceituno, 1989; Garreaud, 2000; Vuille et al., 2000).

\section{Previous geochronological work}

Earlier paleoenvironmental studies of Lake Titicaca generally relied on small numbers of radiocarbon ages from bulk decalcified sediments or macrophyte fragments (Wirrmann and Mourguiart, 1995; Cross et al., 1999, and references therein). These studies presented age models sufficiently accurate for defining Holocene lake-level changes at multi-millennial to millennial timescales. Abbott et al. (1997a,b) used 60 AMS- ${ }^{14} \mathrm{C}$ dates on fish scales, gastropod shells, and macrophytes to precisely define the last 3500 years of lake-level history in the small southern basin of Lake Titicaca. In addition, Abbott et al. (1997a,b) identified a 250 -year ${ }^{14} \mathrm{C}$ hard-water effect for late Holocene sediments of the small basin using ${ }^{210} \mathrm{~Pb}$ geochronometry and paired terrestrial/aquatic radiocarbon ages. The 250-year offset of radiocarbon ages was shown to be nearly constant for the past 3500 years, even during lake lowstands.
Cross et al. (1999) and Baker et al. (2001) defined the timing of late Pleistocene/Holocene lake-level fluctuations using radiocarbon ages derived from bulk decalcified sediment from cores recovered from the deep northern sub-basin of Lake Titicaca. The bulk decalcified organic fraction of most lacustrine sediments is an agglomeration of sub-fractions with multiple origins, variable compositions, and different ages (Mathewes and Westgate, 1980; Nelson et al., 1988). Radiocarbon ages on bulk decalcified sediments represent $\% \mathrm{C}$-weighted integrations of all organic subfractions, minus the small amount of organic matter hydrolyzed during acid decalcification. Comparison of fraction-specific ages can provide more information if macrophytes are absent from the sediment, and ages of several chemically defined organic sub-fractions may provide the best justification for using bulk decalcified ages to support age models. The humin component of sediments is considered to be chemically immobile over the time period used for radiocarbon dating because of its chemical inertness under acidic and alkaline conditions $(<70,000$ years; Hatcher et al., 1985). Radiocarbon ages based on humin residues have not been used extensively for dating lake sediments (Abbott and Stafford, 1996); however, they have been investigated in soils, peats, and organic silts (Cook et al., 1998, and references therein). The humin residue is often determined to be a complex mixture of deposition-age organic matter and older, 
Table 3

Raw and calibrated radiocarbon results for all fractions and the corresponding sediment depths for core site $\mathrm{C} 150 \mathrm{~m}$

\begin{tabular}{|c|c|c|c|c|c|c|c|c|c|}
\hline $\begin{array}{l}\text { Laboratory } \\
\text { accession no. }\end{array}$ & $\begin{array}{l}\text { Depth below } \\
\text { sediment } \\
\text { surface }(\mathrm{cm})\end{array}$ & $\begin{array}{l}\text { Fraction } \\
\text { dated }\end{array}$ & ${ }^{14} \mathrm{C}$ age & Error & $\begin{array}{l}+1 \mathrm{~s} y r \\
\mathrm{BP}\end{array}$ & $\begin{array}{l}\text { Rounded } \\
\text { yr BP } \\
\text { INTCAL98 }\end{array}$ & $\begin{array}{l}-1 \mathrm{~s} y r \\
\mathrm{BP}\end{array}$ & $\begin{array}{l}\text { Coral } \\
\text { calibrated } \\
\text { age yr BP }\end{array}$ & $\begin{array}{l}\text { Model age } \\
\text { yr BP }\end{array}$ \\
\hline OS-21020 & 0.5 & $\mathrm{BD}$ & 0 & 0 & 100 & 50 & 10 & - & $50^{*}$ \\
\hline OS-21021 & 30.0 & $\mathrm{BD}$ & 1490 & 45 & 1260 & 1170 & 1080 & - & $1170 *$ \\
\hline OS-21022 & 60.0 & $\mathrm{BD}$ & 2370 & 40 & 2150 & 2120 & 2010 & - & $2120^{*}$ \\
\hline OS-21023 & 83.0 & $\mathrm{BD}$ & 3490 & 40 & 3470 & 3470 & 3400 & - & $3470^{*}$ \\
\hline OS-21026 & 83.0 & $\mathrm{HU}$ & 3850 & 40 & 3970 & 3890 & 3830 & - & $3890 *$ \\
\hline CAMS-70694 & $107.4^{\dagger}$ & $\mathrm{BD}$ & 4920 & 30 & 5460 & 5400 & 5320 & - & $5400^{*}$ \\
\hline CAMS-70695 & $113.6^{\dagger}$ & $\mathrm{BD}$ & 5190 & 40 & 5710 & 5660 & 5610 & - & $5660^{*}$ \\
\hline CAMS-61618 & 114.3 & $\mathrm{BD}$ & 5170 & 40 & 5660 & 5620 & 5600 & - & $5620^{*}$ \\
\hline CAMS-61619 & 114.3 & HA & 5080 & 40 & 5600 & 5590 & 5490 & - & 5590 \\
\hline CAMS-61620 & 114.3 & $\mathrm{HU}$ & 5120 & 50 & 5650 & 5600 & 5590 & - & $5600^{*}$ \\
\hline CAMS-61639 & 114.3 & MF & 5080 & 50 & 5600 & 5590 & 5490 & - & 5590 \\
\hline CAMS-70696 & $116.9^{\dagger}$ & $\mathrm{BD}$ & 5310 & 40 & 5890 & 5860 & 5740 & - & $5860 *$ \\
\hline CAMS-70697 & $119.9^{\dagger}$ & $\mathrm{BD}$ & 5420 & 30 & 5930 & 5920 & 5910 & - & $5920^{*}$ \\
\hline CAMS-70698 & $125.4^{\dagger}$ & $\mathrm{BD}$ & 5380 & 50 & 5930 & 5910 & 5770 & - & $5910^{*}$ \\
\hline CAMS-70699 & $134.6^{\dagger}$ & $\mathrm{BD}$ & 5390 & 40 & 5930 & 5910 & 5890 & - & $5910^{*}$ \\
\hline CAMS-70700 & $139.9^{\dagger}$ & $\mathrm{BD}$ & 5490 & 40 & 6170 & 5970 & 5930 & - & $5970 *$ \\
\hline OS-20098 & 140.4 & $\mathrm{BD}$ & 5550 & 55 & 6170 & 6100 & 5950 & - & $6100^{*}$ \\
\hline CAMS-70701 & $143.3^{\dagger}$ & $\mathrm{BD}$ & 5570 & 40 & 6170 & 6060 & 5990 & - & $6060 *$ \\
\hline CAMS-70702 & $153.9^{\dagger}$ & $\mathrm{BD}$ & 5900 & 40 & 6470 & 6430 & 6360 & - & $6430 *$ \\
\hline CAMS-61621 & 153.9 & $\mathrm{BD}$ & 6060 & 50 & 6720 & 6640 & 6500 & - & $6640^{*}$ \\
\hline CAMS-61622 & 153.9 & $\mathrm{HU}$ & 6270 & 50 & 6900 & 6820 & 6760 & - & $6820^{*}$ \\
\hline CAMS-61640 & 153.9 & MF & 4580 & 80 & 5030 & 4870 & 4830 & - & $4870^{*}$ \\
\hline CAMS-70703 & $165.9^{\dagger}$ & $\mathrm{BD}$ & 6280 & 40 & 6900 & 6820 & 6760 & - & $6820^{*}$ \\
\hline OS-24101 & 165.9 & $\mathrm{BD}$ & 5820 & 50 & 6410 & 6370 & 6300 & - & $6370 * *$ \\
\hline CAMS-61643 & 172.4 & MF & 5370 & 60 & 5930 & 5910 & 5750 & - & 5910 \\
\hline CAMS-61641 & 178.4 & MF & 5340 & 60 & 5910 & 5890 & 5750 & - & 5890 \\
\hline CAMS-69704 & 191.4 & $\mathrm{HU}^{\S}$ & 6630 & 40 & 7410 & 7290 & 7270 & - & $7290 *$ \\
\hline CAMS-69705 & 191.4 & $\mathrm{HA}^{\S}$ & 6550 & 60 & 7270 & 7250 & 7100 & - & 7250 \\
\hline OS-20094 & 200.4 & $\mathrm{BD}$ & 7240 & 75 & 7930 & 7810 & 7700 & - & $7810^{*}$ \\
\hline CAMS-61623 & 210.0 & $\mathrm{BD}$ & 7730 & 50 & 8360 & 8230 & 8190 & - & $8230^{*}$ \\
\hline CAMS-61624 & 210.0 & HA & 7660 & 60 & 8330 & 8180 & 8170 & - & 8180 \\
\hline CAMS-61625 & 210.0 & $\mathrm{HU}$ & 7800 & 50 & 8390 & 8370 & 8340 & - & $8370^{*}$ \\
\hline CAMS-61626 & 221.9 & $\mathrm{BD}$ & 8430 & 50 & 9260 & 9130 & 9030 & - & $9130^{*}$ \\
\hline CAMS-61627 & 221.9 & HA & 8320 & 40 & 9030 & 9010 & 9000 & - & 9010 \\
\hline CAMS-61628 & 221.9 & $\mathrm{HU}$ & 8490 & 40 & 9400 & 9170 & 9090 & - & $9170^{*}$ \\
\hline CAMS-61642 & 221.9 & MF & 8190 & 40 & 8980 & 8850 & 8640 & - & 8850 \\
\hline OS-24102 & 236.9 & $\mathrm{BD}$ & 8830 & 45 & 9550 & 9540 & 9530 & - & $9540 *$ \\
\hline OS-24103 & 253.9 & $\mathrm{BD}$ & 9340 & 50 & 10,240 & 10,220 & 10,210 & - & $10,220 *$ \\
\hline OS-20095 & 260.4 & $\mathrm{BD}$ & 10,050 & 85 & 11,230 & 11,200 & 11,170 & - & $11,200^{*}$ \\
\hline CAMS-69707 & 263.2 & $\mathrm{HU}^{\S}$ & 9760 & 40 & 11,060 & 10,740 & 10,690 & - & $10,740^{*}$ \\
\hline CAMS-69706 & 263.2 & $\mathrm{HA}^{\S}$ & 9320 & 50 & 10,240 & 10,220 & 10,190 & - & 10,220 \\
\hline CAMS-61629 & 280.2 & $\mathrm{BD}$ & 10,480 & 50 & 12,290 & 11,790 & 11,760 & - & $11,790^{*}$ \\
\hline CAMS-61630 & 280.2 & HA & 10,420 & 50 & 12,100 & 11,820 & 11,660 & - & 11,820 \\
\hline CAMS-61631 & 280.2 & $\mathrm{HU}$ & 10,710 & 50 & 12,790 & 12,490 & 12,180 & - & $12,490 *$ \\
\hline OS-24104 & 281.7 & $\mathrm{BD}$ & 10,400 & 70 & 12,090 & 11,750 & 11,570 & - & $11,750 *$ \\
\hline CAMS-61632 & 303.2 & $\mathrm{BD}$ & 11,030 & 50 & 12,950 & 12,880 & 12,660 & - & 12,880 \\
\hline CAMS-61633 & 303.2 & HA & 10,870 & 40 & 12,880 & 12,730 & 12,430 & - & 12,730 \\
\hline CAMS-61634 & 303.2 & $\mathrm{HU}$ & 11,310 & 50 & 13,150 & 13,020 & 12,990 & - & $13,020^{*}$ \\
\hline OS-24105 & 306.7 & $\mathrm{BD}$ & 10,970 & 55 & 12,930 & 12,860 & 12,640 & - & 12,860 \\
\hline OS-24106 & 314.3 & $\mathrm{BD}$ & 11,250 & 55 & 13,140 & 13,000 & 12,900 & - & 13,000 \\
\hline CAMS-71086 & 329.5 & $\mathrm{HU}$ & 11,530 & 50 & 13,390 & 13,170 & 13,150 & - & $13,170^{*}$ \\
\hline
\end{tabular}


Table 3 (continued)

\begin{tabular}{|c|c|c|c|c|c|c|c|c|c|}
\hline $\begin{array}{l}\text { Laboratory } \\
\text { accession no. }\end{array}$ & $\begin{array}{l}\text { Depth below } \\
\text { sediment } \\
\text { surface }(\mathrm{cm})\end{array}$ & $\begin{array}{l}\text { Fraction } \\
\text { dated }\end{array}$ & ${ }^{14} \mathrm{C}$ age & Error & $\begin{array}{l}+1 \mathrm{~s} y r \\
\mathrm{BP}\end{array}$ & $\begin{array}{l}\text { Rounded } \\
\text { yr BP } \\
\text { INTCAL98 }\end{array}$ & $\begin{array}{l}-1 \mathrm{~s} y \mathrm{r} \\
\mathrm{BP}\end{array}$ & $\begin{array}{l}\text { Coral } \\
\text { calibrated } \\
\text { age yr BP }\end{array}$ & $\begin{array}{l}\text { Model age } \\
\text { yr BP }\end{array}$ \\
\hline CAMS-71087 & 329.5 & HA & 10,940 & 40 & 12,910 & 12,830 & 12,640 & - & 12,830 \\
\hline OS-26860 & 334.8 & $\mathrm{BD}$ & 12,810 & 60 & 15,460 & 14,650 & 14,340 & 15,130 & 15,130 \\
\hline CAMS-71088 & 338.1 & $\mathrm{HU}$ & 12,180 & 60 & 14,080 & 13,940 & 13,820 & - & $13,940^{*}$ \\
\hline CAMS-71089 & 338.1 & HA & 11,250 & 40 & 13,130 & 13,000 & 12,900 & - & 13,000 \\
\hline CAMS-69709 & 345.5 & $\mathrm{HU}^{\S}$ & 11,970 & 50 & 13,840 & 13,810 & 13,500 & - & $13,810^{*}$ \\
\hline CAMS-69708 & 345.5 & $\mathrm{HA}^{\S}$ & 11,670 & 40 & 13,760 & 13,420 & 13,180 & - & 13,420 \\
\hline CAMS-71927 & 353.8 & $\mathrm{HU}$ & 11,220 & 40 & 13,120 & 12,990 & 12,890 & - & $12,990^{*}$ \\
\hline CAMS-61635 & 356.4 & $\mathrm{BD}$ & 14,550 & 50 & 17,390 & 17,140 & 16,890 & 17,270 & 17,270 \\
\hline CAMS-61636 & 356.4 & HA & 13,020 & 50 & 15,580 & 15,410 & 14,500 & 15,390 & 15,390 \\
\hline CAMS-61637 & 356.4 & $\mathrm{HU}$ & 15,420 & 50 & 18,420 & 18,140 & 17,870 & 18,320 & $18,320^{*}$ \\
\hline CAMS-74058 & 364.8 & $\mathrm{HU}$ & 14,030 & 50 & 16,780 & 16,540 & 16,310 & 16,630 & $16,630^{*}$ \\
\hline CAMS-74059 & 364.8 & HA & 12,130 & 70 & 14,060 & 13,840 & 13,670 & - & 13,840 \\
\hline OS-24107 & 367.3 & $\mathrm{BD}$ & 14,350 & 65 & 17,160 & 16,910 & 16,660 & 17,020 & 17,020 \\
\hline CAMS-71928 & 370.3 & $\mathrm{HU}$ & 10,710 & 40 & 12,790 & 12,490 & 12,180 & - & $12,490^{*}$ \\
\hline CAMS-74060 & 372.8 & $\mathrm{HU}$ & 14,080 & 70 & 16,840 & 16,590 & 16,360 & 16,690 & $16,690^{*}$ \\
\hline CAMS-74061 & 372.8 & HA & 12,370 & 120 & 15,140 & 14,110 & 13,850 & - & 14,110 \\
\hline CAMS-71929 & 378.3 & $\mathrm{HU}$ & 10,390 & 40 & 11,950 & 11,720 & 11,580 & - & $11,720^{*}$ \\
\hline CAMS-61638 & 380.1 & $\mathrm{BD}$ & 15,660 & 60 & 18,710 & 18,410 & 18,140 & 18,610 & 18,610 \\
\hline CAMS-74062 & 387.1 & $\mathrm{HU}$ & 14,020 & 50 & 16,760 & 16,530 & 16,300 & 16,620 & $16,620^{*}$ \\
\hline CAMS-71930 & 391.1 & $\mathrm{HU}$ & 13,170 & 60 & 15,770 & 15,550 & 15,120 & 15,570 & $15,570^{*}$ \\
\hline CAMS-71931 & 411.1 & $\mathrm{HU}$ & 14,570 & 60 & 17,420 & 17,160 & 16,910 & 17,290 & $17,290^{*}$ \\
\hline CAMS-74063 & 419.1 & $\mathrm{HU}$ & 13,630 & 50 & 16,310 & 16,080 & 15,850 & 16,140 & $16,140^{*}$ \\
\hline CAMS-74513 & 435.1 & $\mathrm{BD}$ & 16,530 & 50 & 19,730 & 19,410 & 19,110 & 19,660 & 19,660 \\
\hline CAMS-74064 & 435.1 & $\mathrm{HU}$ & 15,330 & 50 & 18,310 & 18,030 & 17,770 & 18,210 & $18,210^{*}$ \\
\hline CAMS-71932 & 436.1 & $\mathrm{HU}$ & 16,090 & 70 & 19,220 & 18,910 & 18,610 & 19,130 & $19,130^{*}$ \\
\hline OS-20096 & 439.1 & $\mathrm{BD}$ & 17,200 & 50 & 20,510 & 20,180 & 19,870 & 20,460 & 20,460 \\
\hline CAMS-74065 & 451.1 & $\mathrm{HU}$ & 14,710 & 50 & 17,580 & 17,320 & 17,070 & 17,460 & $17,460 *$ \\
\hline CAMS-74066 & 459.1 & $\mathrm{HU}$ & 16,450 & 50 & 19,630 & 19,320 & 19,020 & 19,560 & $19,560^{*}$ \\
\hline CAMS-71933 & 496.1 & $\mathrm{HU}$ & 15,190 & 60 & 18,150 & 17,870 & 17,610 & 18,050 & $18,050^{*}$ \\
\hline CAMS-74514 & 509.6 & $\mathrm{BD}$ & 15,440 & 50 & 18,440 & 18,160 & 17,890 & 18,350 & 18,350 \\
\hline CAMS-74067 & 509.6 & $\mathrm{HU}$ & 14,420 & 50 & 17,240 & 16,990 & 16,750 & 17,110 & $17,110^{*}$ \\
\hline OS-20097 & 521.6 & $\mathrm{BD}$ & 18,900 & 170 & 22,540 & 22,140 & 21,760 & 22,460 & 22,460 \\
\hline CAMS-74068 & 525.6 & $\mathrm{HU}$ & 16,400 & 50 & 19,580 & 19,260 & 18,960 & 19,500 & $19,500^{*}$ \\
\hline CAMS-74069 & 525.6 & HA & 11,920 & 100 & 13,830 & 13,690 & 13,460 & - & 13,690 \\
\hline CAMS-74848 & 541.6 & $\mathrm{HU}$ & 16,020 & 60 & 19,130 & 18,830 & 18,540 & 19,050 & $19,050^{*}$ \\
\hline CAMS-74070 & 541.6 & $\mathrm{HA}$ & 11,410 & 70 & 13,180 & 13,150 & 13,020 & - & 13,150 \\
\hline CAMS-74071 & 557.6 & $\mathrm{HU}$ & 15,060 & 50 & 17,990 & 17,720 & 17,470 & 17,890 & $17,890^{*}$ \\
\hline CAMS-74072 & 557.6 & HA & 10,010 & 100 & 11,230 & 11,180 & 11,120 & - & 11,180 \\
\hline CAMS-71934 & 581.6 & $\mathrm{HU}$ & 16,810 & 60 & 20,060 & 19,740 & 19,430 & 19,990 & $19,990^{*}$ \\
\hline CAMS-74515 & 597.6 & $\mathrm{BD}$ & 18,730 & 50 & 22,300 & 21,950 & 21,610 & 22,260 & 22,260 \\
\hline CAMS-74073 & 597.6 & $\mathrm{HU}$ & 17,400 & 60 & 20,740 & 20,410 & 20,090 & 20,700 & $20,700^{*}$ \\
\hline CAMS-74074 & 597.6 & HA & 13,790 & 90 & 16,510 & 16,260 & 16,020 & 16,340 & 16,340 \\
\hline OS-20099 & 686.1 & $\mathrm{BD}$ & 20,900 & 190 & $\mathrm{pc}$ & $\mathrm{pc}$ & $\mathrm{pc}$ & 24,780 & 24,780 \\
\hline CAMS-74516 & 688.1 & $\mathrm{BD}$ & 20,550 & 60 & pc & pc & $\mathrm{pc}$ & 24,370 & 24,370 \\
\hline CAMS-74075 & 688.1 & $\mathrm{HU}$ & 18,720 & 60 & 22,290 & 21,930 & 21,600 & 22,250 & $22,250^{*}$ \\
\hline CAMS-74076 & 688.1 & HA & 14,820 & 70 & 17,720 & 17,450 & 17,190 & 17,600 & 17,600 \\
\hline CAMS-74077 & 784.1 & $\mathrm{HU}$ & 20,440 & 70 & 23,910 & 23,910 & 23,520 & 24,250 & $24,250^{*}$ \\
\hline CAMS-74078 & 784.1 & HA & 15,790 & 90 & 18,870 & 18,560 & 18,270 & 18,770 & 18,770 \\
\hline CAMS-74517 & 828.1 & $\mathrm{BD}$ & 22,800 & 70 & $\mathrm{pc}$ & $\mathrm{pc}$ & $\mathrm{pc}$ & 26,930 & 26,930 \\
\hline CAMS-74079 & 828.1 & $\mathrm{HU}$ & 22,100 & 80 & $\mathrm{pc}$ & $\mathrm{pc}$ & $\mathrm{pc}$ & 26,140 & $26,140^{*}$ \\
\hline CAMS-74080 & 828.1 & HA & 17,530 & 100 & 20,910 & 20,560 & 20,230 & 20,850 & 20,850 \\
\hline
\end{tabular}


Table 3 (continued)

\begin{tabular}{|c|c|c|c|c|c|c|c|c|c|}
\hline $\begin{array}{l}\text { Laboratory } \\
\text { accession no. }\end{array}$ & $\begin{array}{l}\text { Depth below } \\
\text { sediment } \\
\text { surface }(\mathrm{cm})\end{array}$ & $\begin{array}{l}\text { Fraction } \\
\text { dated }\end{array}$ & ${ }^{14} \mathrm{C}$ age & Error & $\begin{array}{l}+1 \mathrm{~s} \mathrm{yr} \\
\text { BP }\end{array}$ & $\begin{array}{l}\text { Rounded } \\
\text { yr BP } \\
\text { INTCAL98 }\end{array}$ & $\begin{array}{l}-1 \mathrm{~s} \mathrm{yr} \\
\mathrm{BP}\end{array}$ & $\begin{array}{l}\text { Coral } \\
\text { calibrated } \\
\text { age yr BP }\end{array}$ & $\begin{array}{l}\text { Model age } \\
\text { yr BP }\end{array}$ \\
\hline OS-10384 & 872.1 & BD & 23,400 & 250 & $\mathrm{pc}$ & $\mathrm{pc}$ & $\mathrm{pc}$ & 27,600 & 27,600 \\
\hline CAMS-74081 & 908.1 & $\mathrm{HU}$ & 21,350 & 80 & $\mathrm{pc}$ & $\mathrm{pc}$ & $\mathrm{pc}$ & 25,290 & $25,290 *$ \\
\hline CAMS-74082 & 908.1 & HA & 17,880 & 100 & 21,320 & 20,970 & 20,630 & 21,260 & 21,260 \\
\hline CAMS-74518 & 983.1 & $\mathrm{BD}$ & 25,990 & 100 & $\mathrm{pc}$ & $\mathrm{pc}$ & $\mathrm{pc}$ & 30,460 & 30,460 \\
\hline CAMS-74083 & 983.1 & $\mathrm{HU}$ & 24,420 & 100 & $\mathrm{pc}$ & $\mathrm{pc}$ & $\mathrm{pc}$ & 28,740 & $28,740^{*}$ \\
\hline CAMS-74084 & 983.1 & HA & 20,140 & 90 & 23,980 & 23,570 & 23,180 & 23,900 & 23,900 \\
\hline CAMS-74085 & 1095.1 & $\mathrm{HU}$ & 24,490 & 100 & $\mathrm{pc}$ & $\mathrm{pc}$ & $\mathrm{pc}$ & 28,810 & $28,810^{*}$ \\
\hline CAMS-74086 & 1095.1 & HA & 21,560 & 160 & $\mathrm{pc}$ & $\mathrm{pc}$ & $\mathrm{pc}$ & 25,530 & 25,530 \\
\hline
\end{tabular}

Because subcentimeter-scale correlation of laminations between the two core sites is possible, the mid-Holocene radiocarbon geochronology developed for the BMC core was imported into the $\mathrm{C} 150 \mathrm{~m}$ radiocarbon geochronology. Radiocarbon ages from the BMC core are reported with the corresponding depth from core site $\mathrm{C} 150 \mathrm{~m}$.

(pc): "pre-calibration", indicating that the radiocarbon date is beyond the range of INTCAL98 calibration routine. The bottom $18.7 \mathrm{~cm}$ of boxcore NE98-4BXB is visibly/geochemically correlative to the top $18.7 \mathrm{~cm}$ of piston core NE98-1PC, thus a complete record of sedimentation is represented for core site $\mathrm{C} 150 \mathrm{~m}$.

* Ages used in age model.

** The large incoherency between this date and dates from nearby intervals in core sites $\mathrm{C} 150 \mathrm{~m}$ and BMC resulted in the exclusion of this date from the age model.

${ }^{\dagger}$ Piston core BMC, recovered in the vicinity of core site C150m (see Fig. 1b), is currently being used for detailed studies of organic biomarkers and mid-Holocene organic/inorganic carbon sedimentation.

${ }^{\S}$ Humin and humate dates from discrete intervals denoted in Fig. 2.

recalcitrant, redeposited material, much of which is potentially locked up in clays (Schoute et al., 1983; Blystad and Selsing, 1989; Lowe, 1991, and references therein).

Under certain circumstances wherein the humin sub-fraction is known to include carbon from older sources, the age of the humate sub-fraction may be more accurate (Head et al., 1989, and references therein). However, the mobility of humates through soil and sediment profiles over geologic time is welldocumented (Wassenaar et al., 1990, and references therein), and the validity of constructing age models using ages derived from humate over those from humin must be critically evaluated for each chronology (Shore et al., 1995). A more detailed geochronological investigation of Lake Titicaca sediment cores (Rowe et al., 2002) showed that, although the Holocene portion of the age model constructed by Baker et al. (2001) is sound, the possibility of post-depositional mobility of certain organic fractions may result in several internally consistent age models for latePleistocene sediments. The present study seeks to refine the chronology of late Quaternary lake-level fluctuations that are intimately linked to fluctuations in moisture transport to the Altiplano and large-scale, long-term shifts in tropical hydrologic balance. Here, we introduce 125 new ${ }^{14} \mathrm{C}$ dates on three different sediment fractions from three cores from the deep basin to improve the overall geochronology and to better characterize late Pleistocene age inaccuracies.

\section{Methods}

Samples for radiocarbon analysis were collected from three core sites $(\mathrm{C} 90 \mathrm{~m}, \mathrm{C} 150 \mathrm{~m}, \mathrm{C} 230 \mathrm{~m})$ (Fig. 1b; Table 1) in the deep, northern sub-basin of the lake. Core sites were chosen to reflect a range in water depth $(90-230 \mathrm{~m})$ corresponding to a range in depositional sub-environment. We focused on dating chemically separable organic fractions because macrofossils were largely absent from our cores. We separated and analyzed the bulk decalcified sediment, humate (base-soluble, acid-insoluble), and humin (acid/base insoluble) sedimentary constituents. Laboratory sample preparation included drying bulk sediments at $60{ }^{\circ} \mathrm{C}$ followed by grinding in a mortar and pestle. Pre-weighed samples were acidified $(1 \mathrm{~N} \mathrm{HCl})$ at $90{ }^{\circ} \mathrm{C}$ to remove carbonate. The resulting bulk decalcified fraction was rinsed with deionized water until neutral. An aliquot of bulk decalcified material was reacted with $1 \mathrm{~N} \mathrm{NaOH}$ at $90{ }^{\circ} \mathrm{C}$ to separate the 
Table 4

Raw and calibrated radiocarbon results for all fractions and the corresponding sediment depths for core site $\mathrm{C} 230 \mathrm{~m}$

\begin{tabular}{|c|c|c|c|c|c|c|c|c|c|}
\hline $\begin{array}{l}\text { Laboratory } \\
\text { accession no. }\end{array}$ & $\begin{array}{l}\text { Depth below } \\
\text { sediment } \\
\text { surface }(\mathrm{cm})\end{array}$ & $\begin{array}{l}\text { Fraction } \\
\text { dated }\end{array}$ & ${ }^{14} \mathrm{C}$ age & Error & $\begin{array}{l}+1 \mathrm{~s} \mathrm{yr} \\
\text { BP }\end{array}$ & $\begin{array}{l}\text { Rounded } \\
\text { yr BP } \\
\text { INTCAL98 } \\
\end{array}$ & $\begin{array}{l}-1 \mathrm{~s} y r \\
\mathrm{BP}\end{array}$ & $\begin{array}{l}\text { Coral } \\
\text { calibrated } \\
\text { age yr BP }\end{array}$ & $\begin{array}{l}\text { Model age } \\
\text { yr BP }\end{array}$ \\
\hline CAMS-71319 & 70.0 & $\mathrm{HU}$ & 3050 & 40 & 2950 & 2910 & 2850 & - & $2910^{*}$ \\
\hline CAMS-71320 & 70.0 & HA & 2790 & 40 & 2740 & 2730 & 2510 & - & 2730 \\
\hline OS-17703 & 83.0 & $\mathrm{BD}$ & 3650 & 30 & 3690 & 3670 & 3590 & - & $3670 *$ \\
\hline CAMS-71321 & 108.5 & $\mathrm{HU}$ & 4790 & 40 & 5310 & 5290 & 5050 & - & $5290^{*}$ \\
\hline CAMS-71322 & 108.5 & HA & 4740 & 40 & 5290 & 5110 & 5040 & - & 5110 \\
\hline CAMS-71323 & 133.5 & $\mathrm{HU}$ & 5420 & 40 & 5980 & 5920 & 5910 & - & $5920 *$ \\
\hline CAMS-71324 & 133.5 & HA & 5350 & 40 & 5910 & 5890 & 5750 & - & 5890 \\
\hline OS-20114 & 140.5 & $\mathrm{BD}$ & 5510 & 45 & 6170 & 5990 & 5940 & - & $5990 *$ \\
\hline OS-20610 & 145.5 & $\mathrm{BD}$ & 6540 & 40 & 7260 & 7250 & 7160 & - & $7250 *$ \\
\hline CAMS-71325 & 163.5 & $\mathrm{HU}$ & 6320 & 40 & 6990 & 6900 & 6810 & - & $6900^{*}$ \\
\hline CAMS-71326 & 163.5 & HA & 6340 & 40 & 7000 & 6910 & 6810 & - & 6910 \\
\hline OS-20611 & 185.5 & $\mathrm{BD}$ & 7750 & 110 & 8390 & 8340 & 8180 & - & $8340 *$ \\
\hline CAMS-69710 & 193.5 & $\mathrm{HU}^{\S}$ & 6720 & 50 & 7430 & 7370 & 7320 & - & $7370^{*}$ \\
\hline CAMS-69711 & 193.5 & $\mathrm{HA}^{\S}$ & 6770 & 50 & 7460 & 7430 & 7340 & - & 7430 \\
\hline OS-26870 & 225.5 & $\mathrm{BD}$ & 7820 & 45 & 8400 & 8380 & 8350 & - & $8380 *$ \\
\hline CAMS-69860 & 238.5 & $\mathrm{HU}$ & 8350 & 40 & 9060 & 9010 & 9010 & - & $9010^{*}$ \\
\hline CAMS-69898 & 238.5 & HA & 8420 & 60 & 9260 & 9090 & 9030 & - & 9090 \\
\hline OS-26871 & 275.0 & $\mathrm{BD}$ & 9120 & 45 & 10,150 & 10,020 & 9870 & - & $10,020^{*}$ \\
\hline CAMS-69861 & 293.0 & $\mathrm{HU}$ & 9870 & 40 & 11,160 & 10,920 & 10,790 & - & $10,920 *$ \\
\hline CAMS-70947 & 293.0 & HA & 10,000 & 40 & 11,200 & 11,180 & 11,170 & - & 11,180 \\
\hline CAMS-69712 & 308.0 & $\mathrm{HU}^{\S}$ & 9980 & 50 & 11,200 & 11,170 & 11,160 & - & $11,170^{*}$ \\
\hline CAMS-69713 & 308.0 & $\mathrm{HA}^{\S}$ & 9720 & 50 & 11,040 & 10,690 & 10,600 & - & 10,690 \\
\hline OS-20612 & 315.0 & $\mathrm{BD}$ & 10,400 & 55 & 12,090 & 11,750 & 11,580 & - & $11,750^{*}$ \\
\hline CAMS-69862 & 358.0 & $\mathrm{HU}$ & 11,040 & 40 & 12,950 & 12,890 & 12,660 & - & $12,890^{*}$ \\
\hline CAMS-70948 & 358.0 & HA & 10,960 & 50 & 12,920 & 12,850 & 12,640 & - & 12,850 \\
\hline OS-26872 & 365.0 & $\mathrm{BD}$ & 11,270 & 55 & 13,140 & 13,010 & 12,910 & - & 13,010 \\
\hline CAMS-71092 & 378.0 & $\mathrm{HU}$ & 12,020 & 40 & 13,850 & 13,820 & 13,600 & - & $13,820 *$ \\
\hline CAMS-71093 & 378.0 & HA & 11,670 & 60 & 13,760 & 13,420 & 13,180 & - & 13,420 \\
\hline CAMS-69863 & 401.5 & $\mathrm{HU}$ & 12,870 & 50 & 15,490 & 14,580 & 14,370 & 15,200 & $15,200^{*}$ \\
\hline CAMS-69899 & 401.5 & HA & 12,350 & 50 & 15,090 & 14,100 & 13,850 & - & 14,100 \\
\hline OS-26873 & 418.5 & $\mathrm{BD}$ & 13,290 & 80 & 15,920 & 15,680 & 15,370 & 15,720 & 15,720 \\
\hline CAMS-69714 & 421.5 & $\mathrm{HU}^{\S}$ & 13,370 & 50 & 16,000 & 15,770 & 15,530 & 15,820 & $15,820 *$ \\
\hline CAMS-69715 & 421.5 & $\mathrm{HA}^{\S}$ & 12,800 & 60 & 15,450 & 14,670 & 14,330 & 15,110 & 15,110 \\
\hline CAMS-71327 & 426.5 & $\mathrm{HU}$ & 14,300 & 50 & 17,090 & 16,850 & 16,610 & 16,960 & $16,960 *$ \\
\hline CAMS-71328 & 426.5 & HA & 13,020 & 40 & 15,580 & 15,410 & 14,500 & 15,390 & 15,390 \\
\hline CAMS-71329 & 431.5 & $\mathrm{HU}$ & 14,700 & 50 & 17,570 & 17,310 & 17,060 & 17,450 & $17,450 *$ \\
\hline CAMS-71330 & 431.5 & HA & 12,960 & 50 & 15,550 & 15,350 & 14,430 & 15,310 & 15,310 \\
\hline CAMS-71393 & 436.5 & $\mathrm{HU}$ & 16,250 & 50 & 19,400 & 19,090 & 18,800 & 19,320 & $19,320 *$ \\
\hline CAMS-71331 & 436.5 & HA & 13,100 & 40 & 15,680 & 15,490 & 14,920 & 15,490 & 15,490 \\
\hline CAMS-69864 & 441.5 & $\mathrm{HU}$ & 16,860 & 40 & 20,110 & 19,790 & 19,490 & 20,050 & $20,050 *$ \\
\hline CAMS-70949 & 441.5 & HA & 13,210 & 40 & 15,810 & 15,580 & 15,220 & 15,620 & 15,620 \\
\hline OS-26874 & 478.5 & $\mathrm{BD}$ & 15,810 & 90 & 18,900 & 18,590 & 18,290 & 18,790 & 18,790 \\
\hline CAMS-69865 & 478.5 & $\mathrm{HU}$ & 18,650 & 50 & 22,200 & 21,850 & 21,520 & 22,170 & $22,170^{*}$ \\
\hline CAMS-69900 & 481.5 & HA & 13,280 & 60 & 15,900 & 15,670 & 15,360 & 15,710 & 15,710 \\
\hline OS-20613 & 514.5 & $\mathrm{BD}$ & 17,450 & 80 & 20,810 & 20,470 & 20,150 & 20,750 & 20,750 \\
\hline CAMS-69866 & 521.5 & $\mathrm{HU}$ & 20,650 & 80 & $\mathrm{pc}$ & $\mathrm{pc}$ & $\mathrm{pc}$ & 24,490 & $24,490 *$ \\
\hline CAMS-69901 & 521.5 & HA & 13,210 & 130 & 15,850 & 15,580 & 15,170 & 15,620 & 15,620 \\
\hline CAMS-69867 & 563.0 & $\mathrm{HU}$ & 18,690 & 60 & 22,250 & 21,900 & 21,560 & 22,220 & $22,220 *$ \\
\hline CAMS-70950 & 563.0 & HA & 14,820 & 210 & 17,800 & 17,450 & 17,110 & 17,600 & 17,600 \\
\hline CAMS-69868 & 598.0 & $\mathrm{HU}$ & 21,230 & 80 & $\mathrm{pc}$ & $\mathrm{pc}$ & $\mathrm{pc}$ & 25,150 & $25,150 *$ \\
\hline
\end{tabular}


Table 4 (continued)

\begin{tabular}{llllrlllll}
\hline $\begin{array}{l}\text { Laboratory } \\
\text { accession no. }\end{array}$ & $\begin{array}{l}\text { Depth below } \\
\text { sediment } \\
\text { surface }(\mathrm{cm})\end{array}$ & $\begin{array}{l}\text { Fraction } \\
\text { dated }\end{array}$ & ${ }^{14} \mathrm{C}$ age & Error & $\begin{array}{l}+1 \mathrm{~s} \mathrm{yr} \\
\mathrm{BP}\end{array}$ & $\begin{array}{l}\text { Rounded } \\
\text { yr BP } \\
\text { INTCAL98 }\end{array}$ & $\begin{array}{l}-1 \mathrm{~s} \text { yr } \\
\text { BP }\end{array}$ & $\begin{array}{l}\text { Coral } \\
\text { calibrated } \\
\text { age yr BP }\end{array}$ & $\begin{array}{l}\text { Model age } \\
\text { yr BP }\end{array}$ \\
\hline CAMS-70951 & 598.0 & HA & 15,400 & 130 & 18,430 & 18,110 & 17,820 & 18,300 & 18,300 \\
CAMS-69869 & 633.0 & HU & 21,320 & 70 & pc & pc & pc & 25,260 & $25,260 *$ \\
CAMS-70952 & 633.0 & HA & 15,590 & 430 & 18,910 & 18,330 & 17,780 & 18,530 & 18,530 \\
CAMS-69870 & 673.0 & HU & 20,690 & 80 & pc & pc & pc & 24,540 & $24,540 *$ \\
CAMS-69902 & 673.0 & HA & 14,340 & 230 & 17,250 & 16,890 & 16,550 & 17,010 & 17,010 \\
CAMS-69871 & 714.0 & HU & 20,750 & 60 & pc & pc & pc & 24,600 & $24,600 *$ \\
CAMS-69903 & 714.0 & HA & 14,430 & 190 & 17,330 & 17,000 & 16,680 & 17,120 & 17,120 \\
CAMS-69872 & 749.0 & HU & 22,680 & 80 & pc & pc & pc & 26,800 & $26,800^{*}$ \\
CAMS-69937 & 749.0 & HA & 15,500 & 550 & 18,930 & 18,230 & 17,550 & 18,420 & 18,420 \\
CAMS-69873 & 789.0 & HU & 22,570 & 90 & pc & pc & pc & 26,670 & $26,670 *$ \\
CAMS-70953 & 789.0 & HA & 17,040 & 100 & 20,340 & 20,000 & 19,670 & 20,270 & 20,270 \\
\hline
\end{tabular}

Because core site $\mathrm{C} 230 \mathrm{~m}$ does not incorporate a box core, the depth below sediment surface represented by the top of $\mathrm{C} 230 \mathrm{~m}$ is estimated by visually and geochemically comparing $\mathrm{C} 230 \mathrm{~m}$ with $\mathrm{C} 150 \mathrm{~m}$.

* Ages used in age model.

${ }^{\S}$ Humin and humate dates from discrete intervals denoted in Fig. 2.

humate and humin fractions. Following repeated additions of base and sequential removal of the humate fraction, the humin residue was acidified (1 $\mathrm{N} \mathrm{HCl}, 90{ }^{\circ} \mathrm{C}$ ) and then rinsed three times with deionized water. The humate fraction was precipitated with concentrated $\mathrm{HCl}$ and rinsed three times with deionized water. Following rinsing, all fractions were vacuum-dried, combusted and graphitized. Samples were analyzed at the Center for Accelerator Mass Spectrometry at Lawrence Livermore National Labo- ratory and at the NOSAMS facility at Woods Hole Oceanographic Institution (Tables 2-4).

\section{Results: comparison of ages from multiple cores}

All bulk decalcified, humate, and humin ages are tabulated in Tables $2-4$. In order to show preliminary inter- and intra-fraction age patterns within and between cores, down-core bulk geochemical records

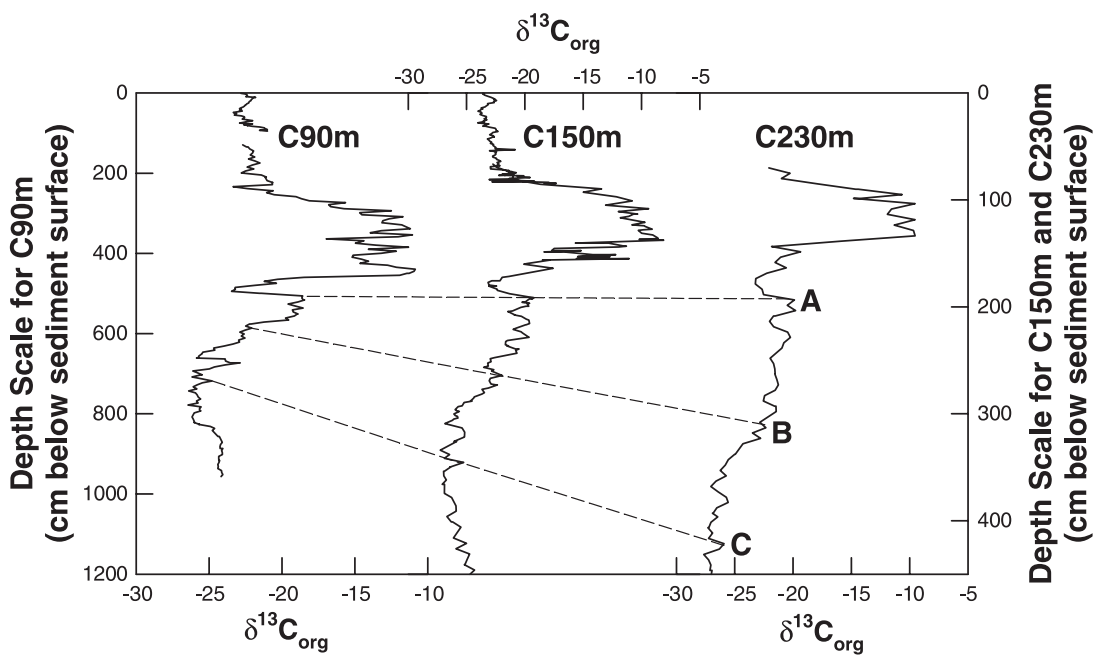

Fig. 2. Down-core stable isotopic records of bulk organic carbon $\left(\delta^{13} \mathrm{C}_{\mathrm{org}}\right)$ from piston cores recovered from the three core sites were used along with $\mathrm{C} / \mathrm{N}$ (not shown) to identify three intervals of similar age. The chosen intervals are connected by dashed lines and denoted "A", "B", and "C". Humate and humin fractions for each interval in each core were radiocarbon dated and are denoted by ' $§$ ' in Tables $2-4$. 
were utilized to identify three discrete, correlative intervals to date in the three piston cores (Fig. 2). Age results for the three intervals, denoted "A", "B", and " $\mathrm{C}$ " in Fig. 2, are indicated by ' $§$ ' in Tables 2-4. The results suggest that within an interval, (1) ages derived from humin have a smaller age range than ages derived from humate, and (2) with only one exception, humate ages are younger than their corresponding humin ages. The preliminary results suggest

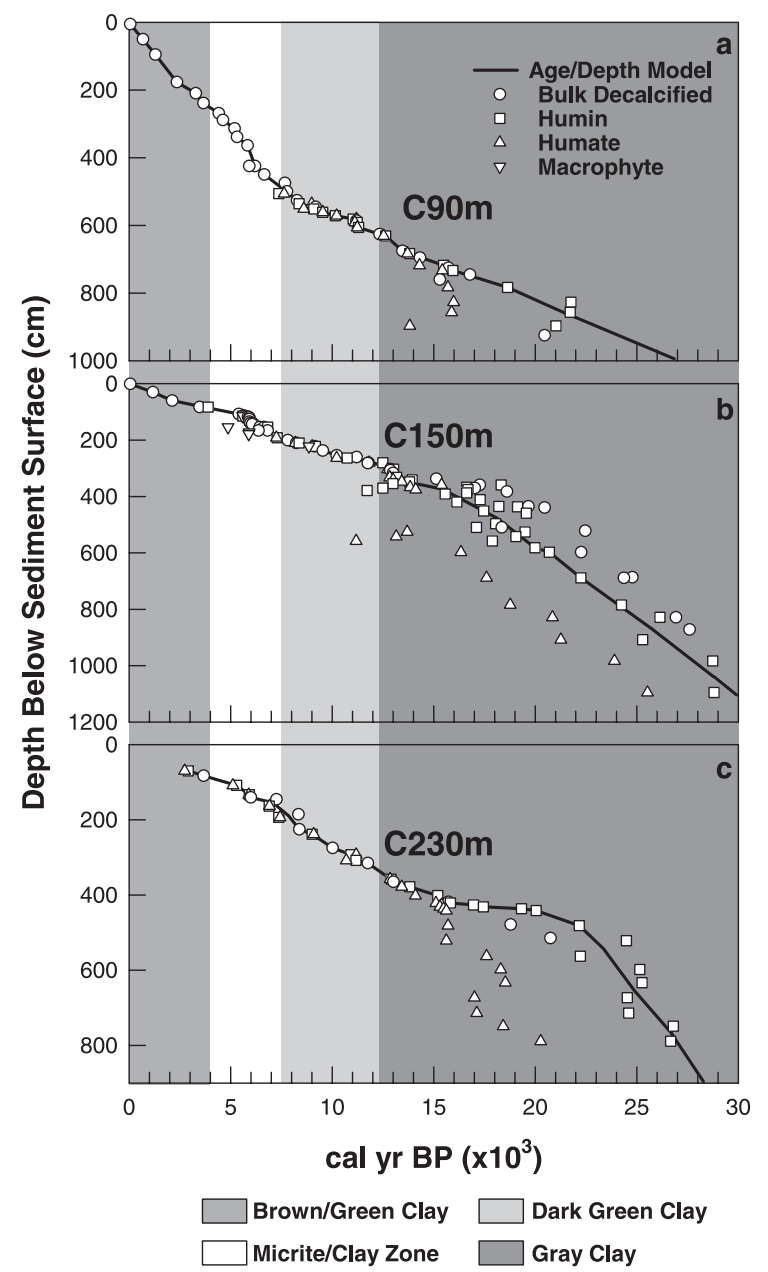

Fig. 3. Down-core bulk decalcified, humate, and humin age results for the three core sites: (a) C90m, (b) C150m, (c) C230m, plotted with respect to calendar age, sediment depth below surface, and a simplified stratigraphy. Solid lines indicate the model used to assign ages to down-core results. Age models are based upon ages from bulk decalcified and humin (humin) sub-fractions in the post-13,500 yr BP (pre-13,500 yr BP) interval. that humin residues give more coherent ages between cores for a given sedimentary interval.

Ages are presented for each core site in the context of sediment depth and stratigraphy (Fig. 3). The age/ depth curves for each fraction in each core are generally consistent down-core, and ages derived from any of the three organic fractions are generally coherent in sediments younger than $13,500 \mathrm{yr}$ BP. In the pre-13,500 yr BP interval, however, ages of the various fractions are divergent, with age offsets as large as 7000 years for a given interval. At sites $\mathrm{C} 90 \mathrm{~m}$ and $\mathrm{C} 230 \mathrm{~m}$, humate ages are consistently youngest, followed by bulk decalcified ages and humin ages (oldest), heretofore denoted the "HA-BD-HU" age pattern. At the intermediate water depth core site, $\mathrm{C} 150 \mathrm{~m}$, humate ages are consistently youngest, followed by humin ages and bulk decalcified ages (oldest), heretofore denoted the "HA-HU-BD" age pattern.

\section{Discussion}

\subsection{Between-core and between-fraction age inhomo- geneity}

Differences in depositional sub-environment create between-core incoherencies in age, geological signature, and proxy record signature that are revealed when comparing multiple down-core records from the same lake. These incoherencies arise from differences in sedimentation rate, bioturbation, pelagic versus littoral input, and detrital input. With the exception of the sample chosen for interval " $\mathrm{C}$ " at core site $\mathrm{C} 150 \mathrm{~m}$, we believe the minor within-fraction age differences for the three discrete intervals in Fig. 2 are predominantly a consequence of the depositional inhomogeneity between the respective core sites. We believe the sample chosen for interval " $\mathrm{C}$ " at core site $\mathrm{C} 150 \mathrm{~m}$ does not represent the same discrete interval chosen for the other two core sites.

In Lake Titicaca sediments, age consistency between different organic fractions in the post-13,500 yr BP sediment interval affirms the use of any or all chemically extractable sub-fractions, including the bulk decalcified fraction, for constructing age/depth models possessing centennial-scale accuracy. This is not the case for the pre-13,500 yr BP interval, within 
which humate, bulk decalcified, and humin ages from a discrete sample interval may span several thousand years (Fig. 3). The HA-BD-HU age pattern observed at core sites $\mathrm{C} 90 \mathrm{~m}$ and $\mathrm{C} 230 \mathrm{~m}$ suggests that these sediments underwent a similar depositional and/or post-depositional history that was different from that of core site $\mathrm{C} 150 \mathrm{~m}$. Mass balance calculations using the organic fractions of $\mathrm{C} 230 \mathrm{~m}$ (not shown) indicate the bulk decalcified fraction is predominantly a mixture of humin and humate sub-fractions. This cannot be the case for core site $\mathrm{C} 150 \mathrm{~m}$, characterized by a bulk decalcified fraction that is older than both humin and humate sub-fractions. The HA-HU-BD age pattern of core site $\mathrm{C} 150 \mathrm{~m}$ implies that the bulk decalcified fraction integrates an older sub-fraction that is hydrolyzed during base extraction but is not re-precipitated during the humate recovery step (addition of concentrated $\mathrm{HCl}$ ). As a consequence, this alkali-mobile, acidsoluble sub-fraction does not contribute to the humate or humin age results. Furthermore, because this subfraction initially hydrolyzes under basic conditions and does not re-precipitate during acidification, it is by definition a fulvic sub-component (e.g., Rashid and King, 1970), which we designate here as the "AlkaliMobile Fulvic" or AMF sub-fraction.

The solubility properties and greater age of the AMF sub-fraction make it inherently different from the humin and humate sub-fractions. The AMF subfraction is not removed during initial sediment decalcification revealing that its origin and/or chemical stability is different from other fulvic acids that are released during the decalcification step. This observation leads us to propose a group of mechanisms that stabilize or "protect" the AMF sub-fraction from hydrolyzing during the initial decalcification step, but permanently destabilize the AMF sub-fraction during the subsequent alkaline treatment. We believe the most suitable set of circumstances under which otherwise-mobile organics are sequestered from solution is through organic-mineral and/or organometallic interactions, or through simple occlusion of organics within mineral pore spaces.

\subsection{Potential mechanisms that stabilize/destabilize the AMF sub-fraction}

We believe the AMF sub-fraction inferred from the $\mathrm{HA}-\mathrm{HU}-\mathrm{BD}$ age pattern at core site $\mathrm{C} 150 \mathrm{~m}$ has its origins through one or several mechanism(s): organic adsorption on mineral surfaces, organometallic interactions, occlusion of organic matter into sealed mineral pore spaces, or interlamellar binding (adsorption/absorption) of fulvic/humic molecules (Schnitzer and Kodama, 1967; Rashid and King, 1970; Greenland, 1971; Rashid, 1971; Rashid et al., 1972; Davis and Gloor, 1981; Davis, 1982; Preston and Riley, 1982; Jardine et al., 1989; Wang and Lee, 1993; Gu et al., 1994, 1995; Kiel et al., 1994; Thimsen and Kiel, 1998; Hedges and Kiel, 1999; Arnarson and Kiel, 2000). Some mechanisms are better at sequestering the AMF sub-fraction than others, and certainly some mechanisms do not uniquely apply to core site $\mathrm{C} 150 \mathrm{~m}$. We believe the origin of the AMF sub-fraction is reasonably explained if eroded clays containing radiocarbondepleted organic matter (fulvic acids) within their interlamellar sites were deposited at core site $\mathrm{C} 150 \mathrm{~m}$ but did not represent a significant depositional component at the other two core sites. The initial decalcification step may extract some of the fulvics held in the interlamellar sites, but much of this subfraction remains immobilized. In addition to the humate fraction, the alkaline hydrolysis liberates most of the interlamellae-bound fulvic fraction, which remains in suspension during the subsequent re-acidification.

Why might core site $\mathrm{C} 150 \mathrm{~m}$ receive clays containing interlamellae-bound carbon and not the other two core sites? A difference in source materials may account for this differential sedimentation pattern. Because of its location in Yunguyo Bay, core site $\mathrm{C} 150 \mathrm{~m}$ may have received a significant portion of its glacial clays from the weathered and glaciated volcanic terrain exposed around the southern portion of Lago Grande. Core site C90m most likely received most of its input from the sedimentary strata (predominantly red sandstones) on the western edge of the lake, and core site $\mathrm{C} 230 \mathrm{~m}$ likely received its glacial inputs directly from the sedimentary strata and granitic complexes exposed in the eastern cordillera. In addition to interlamellar mechanisms, strong mineral surface sorption, organo-metallic complexation, and/ or pore space occlusion of organic constituents potentially explain the reason for the HA-HU-BD age pattern at $\mathrm{C} 150 \mathrm{~m}$, but it is difficult to rationalize why these same mechanisms would not be important at 
core sites $\mathrm{C} 90 \mathrm{~m}$ and $\mathrm{C} 230 \mathrm{~m}$. A detailed investigation of mineral content and provenance would help decipher the sedimentological differences between the core sites.

\subsection{Suitability of the various fractions for construct-} ing age models in the pre-13,500 yr BP interval

Regardless of core location, the youngest extractable organic component in the pre-13,500 yr BP interval is the humate sub-fraction. The younger age of the humate sub-fraction and the significant age difference between the humin and humate components most likely reflect the downward movement of humate through interconnected pore spaces (Wassenaar et al., 1990, and references therein). The solubility of the AMF sub-fraction under alkaline/acidic conditions also characterizes it as a mobile component over geologic time, rendering it unfavorable for age dating purposes. Bulk decalcified ages integrate the humate and AMF sub-fractions, suggesting the bulk decalcified fraction may not be the most suitable fraction to use for constructing age models in sediments prior to $13,500 \mathrm{yr}$ BP. Given this, the humin sub-fraction is apparently the optimal component for age-dating at all core sites in Lake Titicaca. Humin is insoluble over geologic time and the organic-poor Altiplano soils create a low potential for input of old detrital (not interlamellae-bound) carbon from the surrounding basin (Binford et al., 1992; Rodrigo and Wirrmann, 1992). In addition, the bulk $\mathrm{C} / \mathrm{N}$ ratio of glacial age sediments $(\mathrm{C} / \mathrm{N}<6)$ does not reflect a significant terrestrial input of organic matter $(\mathrm{C} /$ $\mathrm{N}>40$ ). Down-core wt. $\% \mathrm{C}_{\text {org }}, \mathrm{C} / \mathrm{N}$, and $\delta^{13} \mathrm{C}_{\text {org }}$ records are plotted to illustrate core-to-core reproducibility within the post-13,500 yr BP interval, and the effectiveness of using the humin fractions for obtaining ages that best represent depositional ages in the pre-13,500 yr BP interval (Fig. 4a-c).

The geochronologies between core sites are slightly diachronous in the middle Holocene interval. In these laminated, carbonate-rich facies, interpreted to represent mid-Holocene lowstand deposits, isotopic and chemical variability at the shallow water core site leads changes at the intermediate and deep water core sites by approximately $500-800$ years. Seismic profiles of the lake sediments (Seltzer et al., 1997) indicate that the mid-Holocene lake level reached $85 \mathrm{~m}$ below modern. This interpretation brings the shallow water core site within $\sim 5 \mathrm{~m}$ of the mid-Holocene shoreline. Given the near-shore setting of core site C90m during the mid-Holocene and the certainty of erosion of exposed lakebed above the core site, organic deposition at the shallow water core site was probably a mixture of "new" organic carbon and slightly older, eroded material. Accordingly, sediments in the mid-

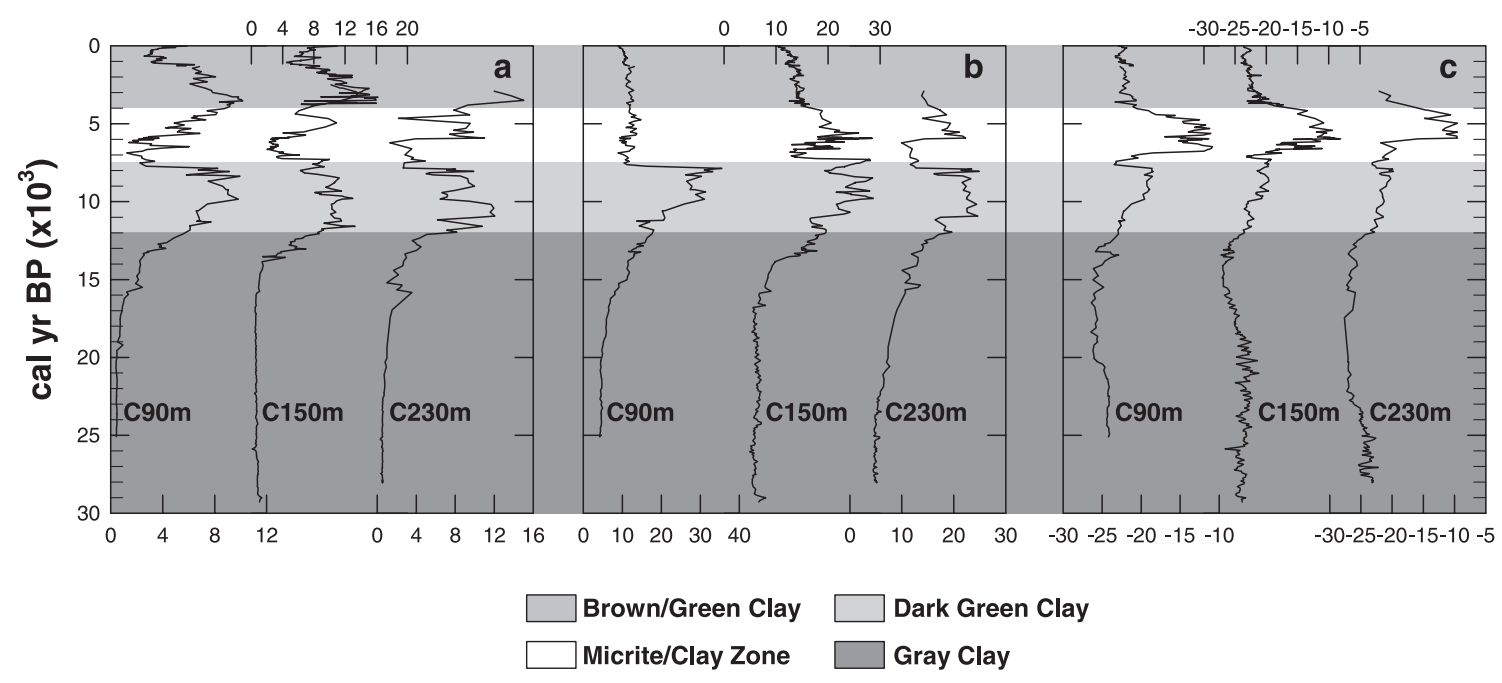

Fig. 4. Down-core (a) wt.\% $\mathrm{C}_{\text {org }}$, (b) $\mathrm{C} / \mathrm{N}$, and (c) $\delta^{13} \mathrm{C}_{\text {org }}$ results for all core sites. A simplified stratigraphy is also shown for each plot. 
Holocene section of the shallow water core site appear slightly older than sediments at the intermediate and deep water core sites, which did not receive significant eroded organic matter.

\subsection{Timing of lake level change and global climate change}

The down-core isotopic composition of bulk organic carbon $\left(\delta^{13} \mathrm{C}_{\text {org }}\right)$ from the deep water core site is plotted with an age model based on eight bulk decalcified and nine humin ages in the post-13,500 yr $\mathrm{BP}$ interval and 16 humin ages from the pre-13,500 yr $\mathrm{BP}$ interval (Fig. 5). $\delta^{13} \mathrm{C}_{\text {org }}$ records relative shifts between lighter isotopic signatures associated with pelagic algae $(\sim-24 \%)$ and heavier isotopic signatures of littoral zone macrophytes $(\sim-12 \pm 4 \%$ ) found in the lake shallows (Cross et al., 1999; Baker et al., 2001; Rowe et al., 2002). Consequently, downcore $\delta^{13} \mathrm{C}_{\text {org }}$ changes with shoreline proximity to core site, and in essence represents late Quaternary changes in the level of Lake Titicaca. Plotted along with the proxy lake-level record are ice-core $\delta^{18} \mathrm{O}$ and $\delta \mathrm{D}$ records from a nearby icecap (Sajama; Thompson et al., 1998), the Greenland Icecap (GISP2; Grootes et al., 1993), and two Antarctic icecaps (Vostok, Jouzel et al., 1996; Taylor Dome, Steig et al., 1998) (Fig. 5). Isotopic changes in the ice cores reflect large-scale changes in hemispheric and global climate.

Fig. 5 illustrates that highstand conditions existed at Lake Titicaca during most of the last glacial episode. According to the $\delta^{13} \mathrm{C}_{\text {org }}$ proxy, a multimillennial regressive phase began during or just prior to the Bølling/Allerød period, and continued into the Pre-Boreal period. The decrease in lake level is probably in response to a weakening of the SASM,

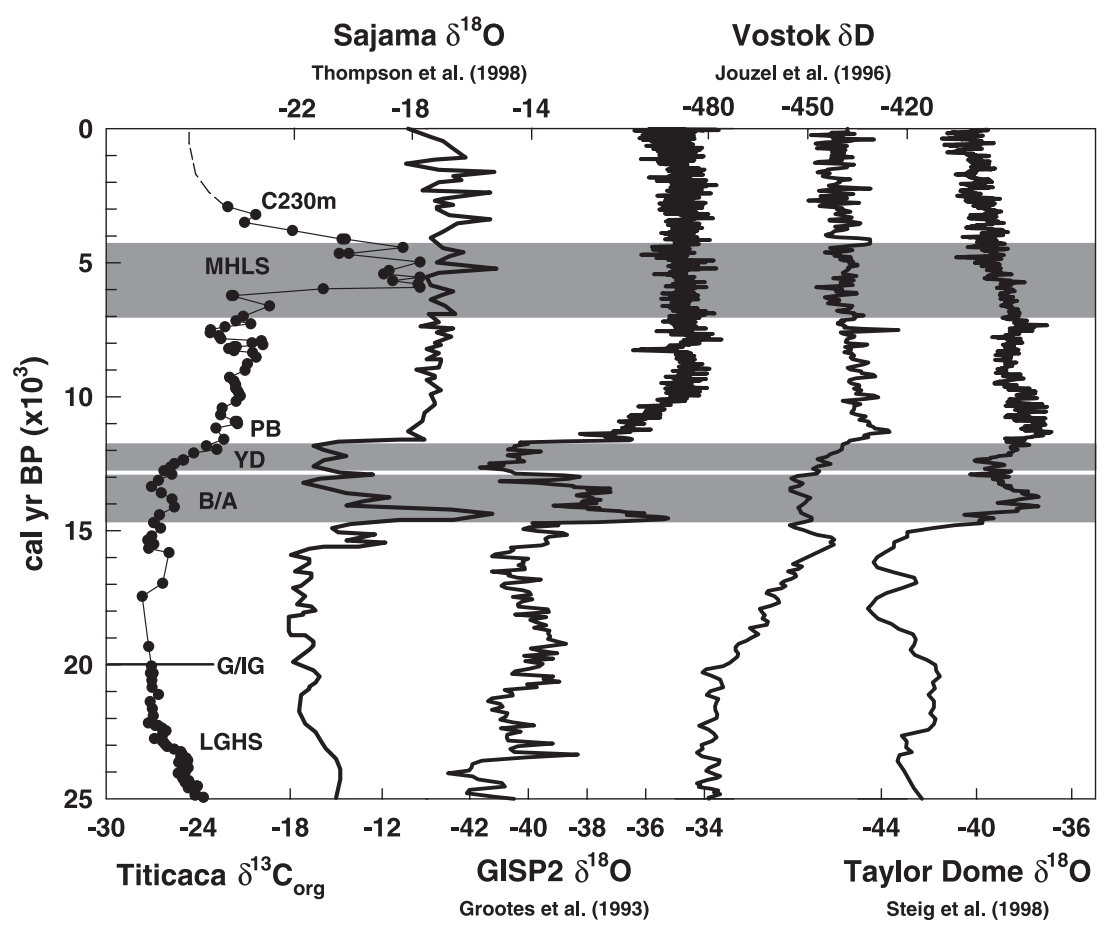

Fig. 5. Down-core $\delta^{13} \mathrm{C}_{\text {org }}$ curve from $\mathrm{C} 230 \mathrm{~m}$ plotted alongside ice-core $\delta^{18} \mathrm{O}$ and $\delta \mathrm{D}$ records from a nearby icecap (Sajama), the Greenland Icecap (GISP2), and two Antarctic icecaps (Vostok, Taylor Dome). The $\delta^{13} \mathrm{C}_{\text {org }}$ curve is interpreted as a record of lake level, by which highstands (lowstands) are characterized by more (less) depleted $\delta^{13} \mathrm{C}_{\text {org }}$, respectively. The last 3000 years of the $\delta^{13} \mathrm{C}_{\text {org }}$ record, represented by the dotted line, is inferred using results from other core sites. LGHS: Last Glacial Highstand; G/IG: Glacial/Interglacial; B/A: Bølling/Allerød; YD: Younger Dryas; PB: Pre-Boreal; MHLS: Mid-Holocene Lowstand. The Glacial/Interglacial boundary denotes when alpine glaciers are thought to have receded on the northern Altiplano, determined by a significant decrease in down-core magnetic susceptibility (Seltzer, personal communications). 
a consequence of decreased summer insolation to the southern hemisphere. The weakening of the SASM across the Pleistocene-Holocene boundary was out of phase with the strengthening West African monsoon, a response to increased northern hemisphere insolation (deMenocal et al., 2000b).

At the submillennial timescale, the $\delta^{13} \mathrm{C}_{\text {org }}$-based lake-level reconstruction of the Bølling/Allerød to PreBoreal period is inconsistent with a previously published diatom-based lake-level record from Lake Titicaca (Baker et al., 2001), although we believe the discordance in interpretations is not a function of different geochronologies, but instead, arises from differences in proxy response to internal lake processes, and to a lesser degree, proxy-specific diagenetic effects, and proxy calibration. The diatom-based lake-level record indicates changes in water depth beginning in the Bølling/Allerød period, but inferences based on the diatom data suggest a series of oscillations in lake level in the period from about 15,000 to $8000 \mathrm{yr}$ BP, in contrast to the more directional pattern evident in the $\delta^{13} \mathrm{C}_{\text {org }}$. Modern data from Lake Titicaca indicate that both $\delta^{13} \mathrm{C}_{\mathrm{org}}$ (Cross et al., 1999; Rowe et al., 2002) and diatom abundance patterns (Tapia et al., manuscript in preparation) show clear relationships to lake depth, and the strength of these relationships supports the use of both proxies as indicators of lake-level change. The differences in pattern of the two proxies during deglaciation likely reflect differences in the thresholds for response to lake-level change and variables affected by lake-level change. Thus, as the elevation of Lake Titicaca fluctuated above and below the outlet level in response to climate variation, the timing and rate of response of the physico-chemical variables that affect diatoms were not identical to the timing and rate of change in the factors that produced changes in $\delta^{13} \mathrm{C}_{\mathrm{org}}$. In any case, it is clear that a distinct lake-level response to insolation change during deglaciation began during or just prior to the Bølling/Allerød period.

Lake level dropped precipitously during the middle Holocene by approximately $85 \mathrm{~m}$, as inferred from ostracode biostratigraphy and seismic stratigraphy (Wirrmann and Mourguiart, 1995, and references therein; Seltzer et al., 1997). The mid-Holocene lowstand (MHLS) at Lake Titicaca is the most obvious climate episode of the last 25,000 years, yet, with the exception of the post- $6000 \mathrm{yr}$ BP variability increase in the $\delta^{18} \mathrm{O}$ profile of the Sajama record, the MHLS is not reflected by substantial changes in the ice-core records. This suggests that ice-core records from the interior of Greenland and Antarctica were largely insensitive to the variables that forced the mid-Holocene regressive phase at Lake Titicaca. However, recent evidence from the Palmer Deep, immediately west of the Antarctic Peninsula, indicates that the middle Holocene was a time of significantly increased surface productivity (Domack et al., 2001), perhaps brought about by a strong teleconnection to sustained El Niño-Southern Oscillation (ENSO) warm mode conditions. The MHLS is thought to be a non-linear coupled response to ENSO and insolation (Martin et al., 1993; Rowe et al., 2002), as it is nearly concurrent with the mid-Holocene re-establishment of ENSO conditions in the equatorial Pacific (Sandweiss et al., 2001, and references therein). The shift toward a strengthened SASM (and weakened West African monsoon) occurred between 5000 and 6000 yr BP. The subsequent transgressive phase, leading to modern lake highstand conditions, began as early as 5000 yr BP.

\section{Conclusions}

The timing of continent-scale and interhemispheric paleoenvironmental changes can only be as accurate as the geochronological underpinnings of the individual records. Often, the weakest aspect of a paleoenvironmental reconstruction is the age control. The results presented here indicate various pitfalls associated with reliance upon a single core site and a single organic fraction for dating purposes. We have established what we believe is the optimal fraction for constructing an age model for Lake Titicaca that most accurately reflects the age of sediment deposition by comparing radiocarbon ages of chemically defined organic fractions from multiple core sites. Radiocarbon ages derived from bulk decalcified sediment, humate, and humin fractions generally agree within standard AMS- ${ }^{14} \mathrm{C}$ errors of $\sim 50-80$ years for sediments deposited after 13,500 yr BP. Internally consistent chronologies can be developed using bulk decalcified sediment and humate fractions. However, on the basis of mobility, we believe these fractions do not yield the most representative ages for deposition 
in the pre-13,500 $\mathrm{yr}$ BP interval. Because of the presumably insignificant input of old detrital carbon (with the exception of mid-Holocene sedimentation to $\mathrm{C} 90 \mathrm{~m}$ ) and the geochemically inert nature of the acid/ alkali-insoluble residue we selected the humin subfraction to establish core chronology in this analysis, especially in pre-13,500 yr BP sediments.

We used down-core geochemical proxy results to define large-scale changes in the level of Lake Titicaca during the past $\sim 29,000$ years. Comparison of the lake-level record with isotopic profiles from ice cores reveals that the middle Holocene lowstand present at Lake Titicaca is absent in polar ice cores and the Sajama ice core. In the Sajama core it marks the onset of more variable $\delta^{18} \mathrm{O}$. Over a multi-millennial time scale the Lake Titicaca record is consistent among the different lake-level proxies, but in finer detail we must further consider how internal lake processes, postdepositional effects, and the limitations of modern site calibration influence the interpretation of individual proxies. In addition to strengthening the Lake Titicaca record for use as a regional paleohydrology index, the geochronological refinement presented here permits stronger paleoclimate comparisons to be established between the central Andes and distant records of global change.

\section{Acknowledgements}

This work was supported by the University of California's Center for Accelerator Mass Spectrometry minigrant, NSF funding to R. Dunbar (ATM9896152) and G. Seltzer (ATM9708258), and a Stanford University McGee Fund grant to $\mathrm{H}$. Rowe. We sincerely thank Matt Grove and Pedro Tapia for field assistance and discussions, and we deeply value the assistance of Brian Frantz and Paula Zermeño (CAMS-LLNL). The coring and post-coring support of James Broda and Parker Hackett (Woods Hole Oceanographic Institution), the general support of Jorge Calvo and Alfredo de La Paz (USAID, Bolivia), and the warm friendship and support of the Ando Family are deeply valued. We also thank Mark Bush (Florida Institute of Technology) for providing several radiocarbon ages from $\mathrm{C} 150 \mathrm{~m}$, and we greatly appreciate the efforts of Ing. Julio Sanjines and Ing. Mario Revollo (Autoridad Autonoma de Lago Titica- ca), Nicolas Catari and Gonzalo Mollenzon. Radiocarbon analyses were performed under the auspices of the U.S. Department of Energy by the University of California's Lawrence Livermore National Laboratory (contract W-7405-Eng-48). We deeply appreciate the editorial comments of Mark Abbott and Lisa Sloan.

\section{References}

Abbott, M.B., Stafford, T.W., 1996. Radiocarbon geochemistry of modern and ancient Arctic lake systems, Baffin Island, Canada. Quat. Res. 45, 300-311.

Abbott, M.B., Binford, M.W., Brenner, M., Kelts, K.R., 1997a. A $3500{ }^{14} \mathrm{C}$ yr high-resolution record of water-level changes in Lake Titicaca, Bolivia/Peru. Quat. Res. 47, 169-180.

Abbott, M.B., Seltzer, G.O., Kelts, K.R., Southon, J., 1997b. Holocene paleohydrology of the tropical Andes from lake records. Quat. Res. 47, 70-80.

Aceituno, P., 1989. On the functioning of the Southern Oscillation in the South American sector: Part II. Upper-air circulation. J. Clim. 2, 341-355.

Arnarson, T.S., Kiel, R.G., 2000. Mechanisms of pore water organic matter and adsorption to montmorillonite. Mar. Chem. 71, 309-320.

Baker, P.A., Seltzer, G.O., Fritz, S.C., Dunbar, R.B., Grove, M.J., Tapia, P.M., Cross, S.L., Rowe, H.D., Broda, J.P., 2001. The history of South American tropical precipitation for the past 25,000 years. Science 291, 640-643.

Bard, E., Arnold, M., Hamelin, B., Tisnerat-Laborde, N., Cabioch, G., 1998. Radiocarbon calibration by means of mass spectrometric ${ }^{230} \mathrm{Th} /{ }^{234} \mathrm{U}$ and ${ }^{14} \mathrm{C}$ ages of corals. An updated database including samples from Barbados, Mururoa, and Tahiti. Radiocarbon 40 (3), 1085-1092.

Binford, M.W., Brenner, M., Engstrom, D.R., 1992. Temporal sedimentation patterns in the nearshore littoral of Lago Huiñaimarca. In: Dejoux, C., Iltis, A. (Eds.), Lake Titicaca. A Synthesis of Limnological Knowledge. Kluwer Academic Publishing, Dordrecht, The Netherlands, pp. 29-39.

Blystad, P., Selsing, L., 1989. Some erroneous radiocarbon dates of lacustrine sediments. Nor. Geol. Tridskr. 69, 201-208.

Cook, G.T., Dugmore, A.J., Shore, J.S., 1998. The influence of pretreatment of humic acid yields and ${ }^{14} \mathrm{C}$ age of Carex peat. Radiocarbon 40 (1), 21-27.

Cross, S.L., Baker, P.A., Seltzer, G.O., Fritz, S.C., Dunbar, R.B., 1999. A new estimate of the Holocene lowstand level of Lake Titicaca, and implications for tropical paleohydrology. Holocene $10,21-32$.

Davis, J.A., 1982. Adsorption of natural dissolved organic matter at the oxide/water interface. Geochim. Cosmochim. Acta 46, $2381-2393$.

Davis, J.A., Gloor, R., 1981. Adsorption of dissolved organics in lake water by aluminum oxide. Effect of molecular weight. Environ. Sci. Technol. 15 (10), 1223-1229. 
deMenocal, P., Ortiz, J., Guilderson, T., Sarnthein, M., 2000a. Coherent high- and low-latitude climate variability during the Holocene warm period. Science 288, 2198-2202.

deMenocal, P., Ortiz, J., Guilderson, T., Adkins, J., Sarnthein, M., Baker, L., Yarusinsky, M., 2000b. Abrupt onset and termination of the African humid period. Rapid climate responses to gradual insolation forcing. Quat. Sci. Rev. 19, 347-361.

Domack, E., Leventer, A., Dunbar, R., Taylor, F., Brachfeld, S., Sjunneskog, C., 2001. Chronology of the Palmer Deep site, Antarctic Peninsula: a Holocene palaeoenvironmental reference for the circum-Antarctic. Holocene 11 (1), 1-9.

Garreaud, R.D., 1999. Multiscale analysis of the summertime precipitation over the central Andes. Mon. Weather Rev. 127, 901-921.

Garreaud, R.D., 2000. Intraseasonal variability of moisture and rainfall over the South American Altiplano. Mon. Weather Rev. 128, 3337-3346.

Greenland, D.J., 1971. Interactions between humic and fulvic acids and clays. Soil Sci. 111, 34-41.

Grootes, P.M., Stuiver, M., White, J.W.C., Johnsen, S., Jouzel, J., 1993. Comparison of oxygen isotope records from the GISP2 and GRIP Greenland ice cores. Nature 366, 552-555.

Gu, B., Schmitt, J., Chen, Z., Liang, L., McCarthy, J.F., 1994. Adsorption and desorption of natural organic matter on iron oxide, Mechanisms and models. Environ. Sci. Technol. 28, $38-46$.

Gu, B., Schmitt, J., Chen, Z., Liang, L., McCarthy, J.F., 1995. Adsorption and desorption of different organic matter fractions on iron oxide. Geochim. Cosmochim. Acta 59, 219-229.

Guilderson, T.P., Fairbanks, R.G., Rubenstone, J.L., 1994. Tropical temperature variations since 20,000 years ago. Modulating interhemispheric climate change. Science 263, 663-665.

Guilderson, T.P., Fairbanks, R.G., Rubenstone, J.L., 2001. Tropical Atlantic coral oxygen isotopes. Glacial-interglacial sea surface temperatures and climate change. Mar. Geol. 172, 75-89.

Hammarlund, D., Aravena, R., Barnekow, L., Buchardt, B., Possnert, G., 1997. Multi-component carbon isotope evidence of early Holocene environmental change and carbon-flow pathways from a hard-water lake in northern Sweden. J. Paleolimnol. 18, 219-233.

Hatcher, P.G., Breger, I.A., Maciel, G.E., Szeverenyi, N.M., 1985. Geochemistry of humin. In: Aiken, G.R., McKnight, D.M., Wershaw, R.L. (Eds.), Humic Substances in Soil, Sediment, and Water. Geochemistry, Isolation, and Characterization. Wiley, New York, NY, pp. 275-302.

Head, M.J., Zhou, W., Zhou, M., 1989. Evaluation of ${ }^{14} \mathrm{C}$ ages of organic fractions of paleosols from loess-paleosol sequences near Xian, China. Radiocarbon 31 (3), 680-696.

Hedges, J.I., Kiel, R.G., 1999. Organic geochemical perspectives on estuarine processes. Sorption reactions and consequences. Mar. Chem. 65, 55-65.

Jardine, P.M., Weber, N.L., McCarthy, J.F., 1989. Mechanisms of dissolved organic carbon adsorption on soil. J. Soil Sci. Soc. Am. 53, 1378-1385.

Jouzel, J., Waelbroeck, C., Malaize, B., Bender, M., Petit, J.R., Stievenard, M., Barkov, N.I., Barnola, J.M., King, T., Kotlyakov, V.M., Lipenkov, V., Lorius, C., Raynaud, D., Ritz, C.,
Sowers, T., 1996. Climatic interpretation of the recently extended Vostok ice records. Clim. Dyn. 12, 513-521.

Kiel, R.G., Montluçon, D.B., Prahl, F.G., Hedges, J.I., 1994. Sorptive preservation of labile organic matter in marine sediments. Nature 370, 549-552.

Lenters, J.D., Cook, K.H., 1999. Summertime precipitation variability over South America. Role of the large-scale circulation. Mon. Weather Rev. 127, 409-431.

Lowe, J.J., 1991. Stratigraphic resolution and radiocarbon dating of Devensian lateglacial sediments. Quat. Proc. 1, 19-25.

Lowe, J.J., Walker, M.J.C., 2000. Radiocarbon dating the last glacial-interglacial transition (CA. 14-9 ka BP) in terrestrial and marine records. The need for new quality assurance protocols. Radiocarbon 40, 53-68.

Martin, L., Fournier, M., Mourguiart, P., Sifeddine, A., Turcq, B., Absy, L.M., Flexor, J.M., 1993. Southern oscillation signal in South America paleoclimatic data of the last 7000 years. Quat. Res. 39, 338-346.

Mathewes, R.W., Westgate, J.A., 1980. Bridge River Tephra. Revised distribution and significance for detecting old carbon errors in radiocarbon dates of limnic sediments in southern British Columbia. Can. J. Earth Sci. 17, 1454-1461.

Monnheim, F., 1956. Beiträge zur klimatologie und hydrologie des Titicacabeckens. Heidelb. Geogr. Arb. p. 152.

Mourguiart, P., 1990. Une approche nouvelle du probleme posé par les reconstrutions des paléoniveaux lacustres: utilisation d'une fontion de transfert basée sur les faunes d'ostracodes. Géodynamique $5,151-166$.

Mourguiart, P., Correge, T., Wirrmann, D., Argollo, J., Montenegro, M.E., Pourchet, M., Carbonel, P., 1998. Holocene paleohydrology of Lake Titicaca estimated from an ostracod-based transfer function. Palaeogeogr. Palaeoclimatol. Palaeoecol. 143, 51-72.

Nelson, R.E., Carter, L.D., Robinson, S.W., 1988. Anomalous radiocarbon ages from a Holocene detrital organic lens in Alaska and their implications for radiocarbon dating and paleoenvironmental reconstructions in the Arctic. Quat. Res. 29, 66-71.

Pilcher, J.R., 1991. Radiocarbon dating for the Quaternary scientist. Quat. Proc. 1, 27-33.

Preston, M.R., Riley, J.P., 1982. The interactions of humic compounds with electrolytes and three clay minerals under simulated estuarine conditions. Estuar. Coast. Shelf Sci. 14, 567-576.

Rashid, M.A., 1971. Role of humic acids of marine origin and their different molecular weight fractions in complexing di- and trivalent metals. Soil Sci. 111, 298-306.

Rashid, M.A., King, L.H., 1970. Major oxygen-containing functional groups present in humic and fulvic fractions isolated from contrasting marine environments. Geochim. Cosmochim. Acta 34, 193-201.

Rashid, M.A., Buckley, D.E., Robertson, K.R., 1972. Interactions of a marine humic acid with clay minerals and a natural sediment. Geoderma 8, 11-27.

Roche, M.A., Bourges, J., Cortes, J., Mattos, R., 1992. Climatology and hydrology of the Lake Titicaca basin. In: Dejoux, C., Iltis, A. (Eds.), Lake Titicaca. A Synthesis of Limnological Knowledge. Kluwer Academic Publishing, Dordrecht, The Netherlands, pp. $63-88$. 
Rodrigo, L.A., Wirrmann, D., 1992. General aspects of present day sedimentation. In: Dejoux, C., Iltis, A. (Eds.), Lake Titicaca. A Synthesis of Limnological Knowledge Kluwer. Academic Publishing, Dordrecht, The Netherlands, pp. 23-28.

Rowe, H.D., Dunbar, R.B., Mucciarone, D.A., Seltzer, G.O., Baker, P.A., Fritz, S.C., 2002. Insolation, moisture balance, and climate change on the South American Altiplano since the last glacial maximum. Clim. Change 52, 175-199.

Sandweiss, D.H., Maasch, K.A., Burger, R.L., Richardson III, J.B., Rollins, H.B., Clement, A., 2001. Variation in Holocene El Niño frequencies: climate records and cultural consequences in ancient Peru. Geology 29, 603-606.

Schnitzer, M., Kodama, H., 1967. Reactions between a podzol fulvic acid and Na-montmorillonite. Proc. Soil Sci. Soc. Am. $31,632-636$.

Schoute, J.F., Mook, M.G., Streurerman, H.J., 1983. Radiocarbon dating of vegetation horizons. In: Mook, W.G., Waterbolk, H.T. (Eds.), Groningen Conference on ${ }^{14} \mathrm{C}$ and Archaeology. Proc. PACT Publ. 8. Université Catholique de Louvain, Louvain-laNueve, Belgium, pp. 295-311.

Seltzer, G.O., Baker, P.A., Cross, S., Dunbar, R.B., Fritz, S., 1997. High-resolution seismic reflection profiles from Lake Titicaca, Peru-Bolivia. Evidence for Holocene aridity in the tropical Andes. Geology 26, 167-170.

Shore, J.S., Bartley, D.D., Harkness, D.D., 1995. Problems encountered with the ${ }^{14} \mathrm{C}$ dating of peat. Quat. Sci. Rev. 14, 373-383.

Shotton, F.W., 1972. An example of hard water error in radiocarbon dating of vegetable matter. Nature 240, 460-461.

Steig, E.J., Brook, E.J., White, J.W.C., Sucher, C.M., Bender, M.L., Lehman, S.J., Morse, D.L., Waddington, E.D., Clow, G.D., 1998. Synchronous climate changes in Antarctica and the North Atlantic. Science 282, 92-95.

Stuiver, M., Polach, H.A., 1977. Discussion. Reporting of ${ }^{14} \mathrm{C}$ data. Radiocarbon 19, 355-363.

Stuiver, M., Reimer, P.J., 1993. Extended ${ }^{14} \mathrm{C}$ database and revised CALIB radiocarbon calibration program. Radiocarbon $35,215-230$.

Stuiver, M., Reimer, P.J., Bard, E., Beck, J.W., Burr, G.S., Hughen, K.A., Kromer, B., McCormac, F.G., v. d. Plicht, J., Spurk, M., 1998. INTCAL98 radiocarbon age calibration 24,000-0 cal BP. Radiocarbon 40, 1041-1083.

Tapia, P., Fritz, S.C., Baker, P.A., Seltzer, G.O., manuscript in preparation. A late-Quaternary diatom record of tropical climate history from Lake Titicaca, Bolivia/Peru. Palaeogeogr., Palaeoclimatol., Palaeoecol.
Thimsen, C.A., Kiel, R.G., 1998. Potential interactions between sedimentary dissolved organic matter and mineral surfaces. Mar. Chem. 62, 65-76.

Thompson, L.E., Davis, M.E., Mosley-Thompson, E., Sowers, T.A., Henderson, K.A., Zagorodov, V.S., Lin, P.-N., Mikhalenko, V.N., Campen, R.K., Bolzan, J.F., Cole-Dai, J., Francou, B., 1998. A 25,000-year tropical climate history from Bolivian ice cores. Science 282, 1858-1864.

Van Klinken, G.J., Hedges, R.E.M., 1998. Chemistry strategies for organic ${ }^{14} \mathrm{C}$ samples. Radiocarbon 40 (1), 51-56.

Vuille, M., 1999. Atmospheric circulation over the Bolivian Altiplano during dry and wet periods and extreme phases of the Southern Oscillation. Int. J. Clim. 19, 1579-1600.

Vuille, M., Bradley, R.S., Keimig, F., 2000. Interannual climate variability in the central Andes and their relation to tropical Pacific and Atlantic forcing. J. Geophys. Res. 105, 12.447-12.460.

Wang, X.-C., Lee, C., 1993. Adsorption and desorption of aliphatic amines, amino acids, and acetate by clay minerals and marine sediments. Mar. Chem. 44, 1-23.

Wassenaar, L., Aravena, R., Fritz, P., Barker, J., 1990. Isotopic composition $\left({ }^{13} \mathrm{C},{ }^{14} \mathrm{C},{ }^{2} \mathrm{H}\right)$ and geochemistry of aquatic humic substances from groundwater. Org. Geochem. 15 (4), 383-396.

Wirrmann, J.P., 1992. Morphology and bathymetry. In: Dejoux, C., Iltis, A. (Eds.), Lake Titicaca. A Synthesis of Limnological Knowledge. Kluwer Academic Publishing, Dordrecht, The Netherlands, pp. 16-23.

Wirrmann, D., Fernando De Oliveira Almeida, L., 1987. Low Holocene level (7700 to 3650 years ago) of Lake Titicaca (Bolivia). Palaeogeogr. Palaeoclimatol. Palaeoecol. 59, 315-323.

Wirrmann, D., Mourguiart, P., 1995. Late Quaternary spatio-temporal limnological variations in the Altiplano of Bolivia and Peru. Quat. Res. 43, 344-354.

Wirrmann, D., Mourguiart, P., Fernando de Oliveira Almeida, L., 1990. Holocene sedimentology and ostracods distribution in Lake Titicaca-paleohydrological interpretations. In: Rabassa, J. (Ed.), Quaternary of South America and Antarctic Peninsula, vol. 6. A.A. Balkema, Rotterdam, pp. 89-129.

Wirrmann, J.P., Ybert, J.P., Mourguiart, P., 1992. A 20,000 years paleohydrological record from Lake Titicaca. In: Dejoux, C., Iltis, A. (Eds.), Lake Titicaca. A Synthesis of Limnological Knowledge. Kluwer Academic Publishing, Dordrecht, The Netherlands, pp. 40-48.

Zhou, J., Lau, K.-M., 1998. Does a monsoon climate exist over South America? J. Clim. 11, 1020-1040. 